In preparation for The Astrophysical Journal

\title{
Searching for Narrow Emission Lines in X-ray Spectra: Computation and Methods
}

\author{
Taeyoung Park ${ }^{1}$, David A. van Dyk ${ }^{2}$, Aneta Siemiginowska ${ }^{3}$ \\ ${ }^{1}$ Department of Statistics \\ University of Pittsburgh \\ 4200 Fifth Avenue, Pittsburgh, PA 15120 \\ tpark@pitt.edu \\ ${ }^{2}$ Department of Statistics \\ University of California, \\ 364 ICS Bldg One, Irvine, CA 92697-1250 \\ dvd@ics.uci.edu \\ ${ }^{3}$ Smithsonian Astrophysical Observatory, \\ 60 Garden Street, Cambridge, MA 02138 \\ aneta@head.cfa.harvard.edu
}

\begin{abstract}
The detection and quantification of narrow emission lines in X-ray spectra is a challenging statistical task. The Poisson nature of the photon counts leads to local random fluctuations in the observed spectrum that often results in excess emission in a narrow band of energy resembling a weak narrow line. From a formal statistical perspective, this leads to a (sometimes highly) multimodal likelihood. Many standard statistical procedures are based on (asymptotic) Gaussian approximations to the likelihood and simply cannot be used in such settings. Bayesian methods offer a more direct paradigm for accounting for such complicated likelihood functions but even here multimodal likelihoods pose significant computational challenges. The new Markov chain Monte Carlo (MCMC) methods developed in van Dyk \& Park (2008) and Park \& van Dyk (2008), however, are able to fully explore the complex posterior distribution of the location of a narrow line, and thus provide valid statistical inference. Even with these computational tools, standard statistical quantities such as means and standard deviations cannot adequately summarize inference and standard testing procedures
\end{abstract}


cannot be used to test for emission lines. In this paper, we use new efficient MCMC algorithms to fit the location of narrow emission lines, we develop new statistical strategies for summarizing highly multimodal distributions and quantifying valid statistical inference, and we extend the method of posterior predictive p-values proposed by Protassov et al. (2002) to test for the presence of narrow emission lines in X-ray spectra. We illustrate and validate our methods using simulation studies and apply them to the Chandra observations of the high redshift quasar PG1634+706.

Subject headings: methods: statistical - quasars: emission lines

\section{Introduction}

\subsection{Scientific Background}

Modern X-ray observations show complex structures in both the spatial and spectral domains of various astrophysical sources. Nonetheless, active galalactic nuclei (AGN) including quasars' nuclei remain spatially unresolved even with the highest-resolution X-ray telescopes. Most of their energy is released within the unresolved core, and only spectral and timing information is available to study the nature of the X-ray emission. Generally speaking, emission and absorption lines constitute an important part of the X-ray spectrum in that they can provide information as to the state of plasma. One of the goals of X-ray data analysis is to understand the components present in the spectrum, and to obtain information about the emission and absorption features, as well as their locations and relation to the primary quasar emission. The detection of weak lines in noisy spectra is the main statistical problem in such analyses: Is a bump observed in the spectrum related to a real emission line or is it simply an artifact of the Poissonian noise?

Although quasars' X-ray spectra are usually featureless as expected based on the Comptonization process (see for example Markoff et al. 2005; Sobolewska et al. 2004; Sikora et al. 1997), an important X-ray emission feature identified in AGN and quasars spectra is the iron $\mathrm{K}$ emission line (see recent review by Miller 2007). Determining the origin and the nature of this line is one of main issues in AGN and quasar research. This line is thought to come directly from illuminated accretion flow as a fluorescent process (Fabian 2006). The location of the line in the spectrum indicates the ionization state of iron in the emitting plasma, while

the width of the line tells us the velocity of the plasma (Fabian 2006). The iron line provides a direct probe of the innermost regions of accretion flow and matter in close vicinity of a black hole. 
Absorption features associated with the outflowing matter (warm wind, partial covering absorber) have also been observed in recent X-ray observations (Gallagher et al. 2002; Chartas et al. 2002; Pounds \& Reeves 2007). Although the location and width of absorption lines provide information as to the velocity of the absorber and its distance from the quasar, this article focuses on statistical issues in fitting the spectral location of narrow emission lines, i.e., identifying the ionization state.

There are two parts to the Fe-K-alpha emission line observed in AGN Yaqoob et al. 2001a): one is a broad component thought to be a signature of a relativistic motion in the innermost regions of an accretion flow; the other is a narrow component that is a result of a reflection off the material at larger distances from the central black hole. A detection of the broad component is challenging as it requires a spectral coverage over a large energy range, so the continuum emission is well determined and the broad line can be separated (Reeves et al. 2006). The relativistic line profile is broad and skewed, and two strong peaks of the emission line that originates in a relativistic disk can be prominent and narrow. While the full profile of the broad line may not be easily separable from the continuum, these two peaks may provide a signature for this line in the X-ray spectrum. The broad Fe-line gives an important diagnostic of the gas motion and can be used to determine the spin of a black hole (Miller 2006); see also an alternative model for the "red wing" component by Miller et al. (2008). The narrow component of the line gives diagnostics of the matter outside the accretion disk and conditions at larger distances from the black hole; see Fe-line Baldwin effect discussion in Jiang et al. (2006). Both line components are variable and the line may "disappear" from the spectrum (Yaqoob et al. 2001b).

The spectral resolution of X-ray CCD detectors (for example 100-200 eV in ACIS on Chandra or EPIC on XMM-Newton) is relatively low with respect to the predicted width of narrow $\left(<5000-30,000 \mathrm{~km} \mathrm{~s}^{-1}\right)$ emission or absorption lines in AGN and quasars. Observations with grating instruments (RGS or HEG) can provide high resolution X-ray spectra, but the effective area of the present X-ray telescopes is too low for efficient AGN detections, and only a handful of bright low redshift sources have been observed with gratings to date (Yaqoob 2007). Therefore mainly the X-ray CCD spectra of lower resolution are used to study large samples of AGN and quasars (see for example Guainazzi et al. 2006; Page et al. 2005; Jiménez-Bailón et al. 2005).

Using these relatively low resolution X-ray detectors, the Fe-K-alpha emission line can be narrow enough to be contained entirely in a single detector bin. In some cases (for example in Chandra) the line may occupy a few bins. In this article we focus on the statistical problem of fitting the spectral location of an emission line or a set of emission lines that are narrow. This is a common objective in high-energy analyses, but as we shall discuss fitting 
these relatively narrow features poses significant statistical challenges. In particular we find evidence that using line profiles that are narrower than we actually expect the emission line to be can improve the statistical properties of the fitted emission line location.

\subsection{X-Ray Spectral Analysis}

X-ray spectra, such as those available with the Chandra X-ray Observatory carry much information as to the quasar's physics. Taking advantage of the spectral capacity of such instruments, however, requires careful statistical analysis. For example, the resolution of such instruments corresponds to a fine discretization of the energy spectrum. As a result, we expect a low number of counts in each bin of the X-ray spectrum. Such low-count data make the Gaussian assumptions that are inherent in traditional minimum $\chi^{2}$ fitting inappropriate. A better strategy, which we employ, explicitly models photon arrivals as an inhomogeneous Poisson process (van Dyk et al. 2001). In addition, data are subject to a number of processes that significantly degrade the source counts, e.g., the absorption, non-constant effective area, blurring of photons' energy, background contamination, and photon pile-up. Thus, we employ statistical models that directly account for these aspects of data collection. In particular, we design a highly structured multilevel spectral model with components for both the data collection processes and the complex spectral structures of the sources themselves. In this highly structured spectral model, a Bayesian perspective renders straightforward methods that can handle the complexity of Chandra data (van Dyk et al. 2001; van Dyk \& Kang 2004; van Dyk et al. 2006). As we shall illustrate, these methods allow us to use low-count data, to search for the location of a narrow spectral line, to investigate its location's uncertainty, and to construct statistical tests that measure the evidence in the data for including the spectral line in the source model.

\subsection{A Statistical Model for the Spectrum}

The energy spectrum can be separated into two basic parts: a set of continuum terms and a set of several emission linesa. We begin with a standard spectral model that accounts for a single continuum term along with several spectral lines. Throughout this paper, we use $\theta$ as a general representation of model parameters in the spectral model. The components of $\theta=\left(\theta^{C}, \theta^{L}, \theta^{A}, \theta^{B}\right)$ represent the collection of parameters for the Continuum, (emission)

\footnotetext{
aThe model can be extended to account for absorption lines, but in this paper we focus on additive features such as emission lines.
} 
Lines, Absorption, and Background contamination, respectively. (Notice that the roman letters in the superscripts serve as a mnemonic for these four processes.) Because the Xray emission is measured by counting the arriving photons, we model the expected Poisson counts in energy bin $j \in \mathcal{J}$, where $\mathcal{J}$ is the set of energy bins, as

$$
\Lambda_{j}(\theta)=\Delta_{j} f\left(\theta^{C}, E_{j}\right)+\sum_{k=1}^{K} \lambda_{k} \pi_{j}\left(\mu_{k}, \nu_{k}\right),
$$

where $\Delta_{j}$ and $E_{j}$ are the width and mean energy of bin $j, f\left(\theta^{C}, E_{j}\right)$ is the expected counts per unit energy due to the continuum term at energy $E_{j}, \theta^{C}$ is the set of free parameters in the continuum model, $K$ is the number of emission lines, $\lambda_{k}$ is the expected counts due to the emission line $k$, and $\pi_{j}\left(\mu_{k}, \nu_{k}\right)$ is the proportion of an emission line centered at energy $\mu_{k}$ and with width $\nu_{k}$ that falls into bin $j$. There are a number of smooth parametric forms to describe the continuum in some bounded energy range; in this article we parameterize the continuum term $f$ as a power law, i.e., $f\left(\theta^{C}, E_{j}\right)=\alpha^{C} E_{j}^{-\beta^{C}}$ where $\alpha^{C}$ and $\beta^{C}$ represent the normalization and photon index, respectively. The emission lines can be modeled via the proportions $\pi_{j}\left(\mu_{k}, \nu_{k}\right)$ using narrow Gaussian distributions, Lorentzian distributions, or delta functions; the counts due to the emission line are distributed among the bins according to these proportions. While the Gaussian or Lorentzian function parameterizes an emission line in terms of center and width, the center is the only free parameter with a delta function; the width of the delta function is effectively the width of the energy bin in which it resides.

While the model in Equation 1 is of primary scientific interest, a more complex statistical model is needed to address the data collection processes mentioned in $\$ 1.2$. We use the term statistical model to refer to the model that combines the source or astrophysical model with a model for the stochastic processes involved in data collection and recording. Thus, in addition to the source model, the statistical model describes such processes as instrument response and background contamination. Specifically, to account for the data collection processes, Equation 1 is modified via

$$
\Xi_{l}(\theta)=\sum_{j \in \mathcal{J}} M_{l j} \Lambda_{j}(\theta) d_{j} u\left(\theta^{A}, E_{j}\right)+\theta_{l}^{B}
$$

where $\Xi_{l}(\theta)$ is the expected observed Poisson counts in detector channel $l \in \mathcal{L}, \mathcal{L}$ is the set of detector channels, $M_{l j}$ is the probability that a photon that arrives with energy corresponding to bin $j$ is recorded in detector channel $l$ (i.e., $\mathbf{M}=\left\{M_{l j}\right\}$ is the so-called redistribution matrix or RMF commonly used in X-ray analysis), $d_{j}$ is the effective area (i.e., ARF, a calibration file associated with the X-ray observation) of bin $j, u\left(\theta^{A}, E_{j}\right)$ is the probability that a photon with energy $E_{j}$ is not absorbed, $\theta^{A}$ is the collection of parameters for absorption, and $\theta_{l}^{B}$ is a Poisson intensity of the background counts in channel $l$. While the scatter probability $M_{l j}$ and the effective area $d_{j}$ are presumed known from calibration, the absorption 
probability is parameterized using a smooth function; see van Dyk \& Hans (2002) for details. To quantify background contamination, a second data set is collected that is assumed to consist only of background counts; the background photon arrivals are also modeled as an inhomogeneous Poisson process.

\subsection{Difficulty with Identifying Narrow Emission Lines}

Unfortunately, the statistical methods and algorithms developed in van Dyk et al. (2001) cannot be directly applied to fitting narrow emission lines. There are three obstacles that must be overcome in order to extend Bayesian highly structured models to spectra containing narrow lines. In particular, we must develop (1) new computational algorithms, (2) statistical summaries and methods for inference under highly multimodal posterior distributions, and (3) statistical tests that allow us to quantify the statistical support in the data for including an emission line or lines in the model. Our main objective in this paper is to extend the methods of van Dyk et al. (2001) in these three directions, and to evaluate and illustrate our proposals. Here we discuss each of these challenges in detail.

Challenge 1: Statistical Computation. Fitting the location of narrow lines requires new and more sophisticated computational techniques than those developed by van Dyk et al. (2001). Indeed, the algorithms that we develop require a new theoretical framework for statistical computation: they are not examples of any existing algorithm with known properties. Although the details of this generalization are well beyond the scope of this article, we can offer a heuristic description; a more detailed description is given in Appendix A. Readers who are interested in the necessary theoretical development of the statistical computation techniques are directed to van Dyk \& Park (2004, 2008) and Park \& van Dyk (2008).

The algorithms used by van Dyk et al. (2001) to fit the structured Bayesian model described in $\$ 1.3$ are based on the probabilistic properties of the statistical models. For example, the parameters of a Gaussian line profile can be fit by iteratively attributing a subset of the observed photons to the line profile and using the mean and variance of these photon energies to update the center and width of the line profile. The updated parameters of the line profile are used to again attribute a subset of the photons to the line, i.e., to stochastically select a subset of the photons that are likely to have arisen out of the physical processes at the source corresponding to the emission line. These algorithms are typically very stable. For example, they only return statistically meaningful parameters because the algorithms themselves mimic the probabilistic characteristics of the statistical model. The family of Expectation/Maximization (EM) algorithms (Dempster et al. 1977) and Markov 
chain Monte Carlo (MCMC) methods such as the Gibbs sampler (Geman \& Geman 1984) are the examples of statistical algorithms of this sort.

A drawback of these algorithms is that in some situations they can be slow to converge. When fitting the location of a Gaussian emission line, for example, the location is updated more slowly if the line profile is narrower. This is because only photons with energies very close to the current value of the line location can be attributed to the line. Updating the line location with the mean of the energies of these photons cannot result in a large change in the emission line location. The situation becomes chronic when a delta function is used to model the line profile: The line location parameter sticks at its starting value throughout the iteration.

It is to circumvent this difficulty that we develop both new EM-type algorithms (van Dyk \& Park 2004) and new MCMC samplers specially tailored for fitting narrow lines. Our new samplers are motivated by the Gibbs sampler, but constitute a non-trivial generalization of Gibbs sampling known as partially collapsed Gibbs sampling (van Dyk \& Park 2008; Park \& van Dyk 2008); see Appendix A. Our updated versions of both classes of algorithms are able to fit narrow lines by avoiding the attribution of photons to the emission line during the iteration. Such algorithms tend to require fewer iterations to converge regardless of the width of the emission line. Because they involve additional evaluation of quantities evolving the large dimensional redistribution matrix, $M$, however, each iteration of these algorithms can be significantly more costly in terms of computing time. A full investigation of the relative merit of the algorithms and a description of how the computational trade-offs can be played to derive optimal algorithms are beyond the scope of this paper. Except in Appendix A, we do not discuss the details of the algorithms further in this article; interested readers are directed to van Dyk \& Park (2004, 2008) and Park \& van Dyk (2008).

Challenge 2: Multimodal Likelihoods. The likelihood function for the emission line location(s) is highly multimodal. Each mode corresponds to a different relatively likely location for an emission line or a set of emission lines. Standard statistical techniques such as computing the estimates of the line locations with their associated error bars or confidence intervals implicitly assume that the likelihood function is unimodal and bell shaped. Because this assumption is clearly and dramatically violated, these standard summary statistics are unreliable and inadequate.

Unfortunately, there are no readily available and generally applicable simple statistical summaries to handle highly multimodal likelihoods. Instead we must develop summaries that are tailored to the specific scientific goals in a given analysis. Because general strategies for dealing with multimodal likelihood functions are little known to astronomers and specific 
strategies for dealing with multimodal likelihood functions for the location of narrow spectral lines do not exist, one of the primary goals of this article is to develop and illustrate these methods.

A fully Bayesian analysis of our spectral model with narrow emission lines is computationally demanding, even with our new algorithms. Thus, we develop techniques that are much quicker and give similar results for the location of emission lines. These methods based on the so-called profile posterior distribution do not stand on as firm of a theoretical footing as a fully Bayesian analysis, but are much quicker and thus better suited for exploratory data analysis. The profile posterior distribution along with our exploratory methods are fully described and compared with the more sophisticated Bayesian analysis.

Challenge 3: Testing for the Presence of Narrow Lines. In addition to fitting the location of one or more emission lines, we often would like to perform a formal test for the inclusion of the emission lines in the statistical model. That is, we would like to quantify the evidence in a potentially sparse data set for a particular emission line in the source.

Testing for a spectral line is an example of a notoriously difficult statistical problem in which the standard theory does not apply. There are two basic technical problems. First, the simpler model that does not include a particular emission line is on the boundary of the larger model that does include the line. That is, the intensity parameter of an emission line is zero under the simpler model and cannot be negative under the larger model. An even more fundamental problem occurs if either the line location or width is fit, because these parameters have no value under the simpler model. The behavior (i.e., sampling distribution) of the likelihood ratio test statistic under the simpler model is not well understood and cannot be assumed to follow the standard $\chi^{2}$ distribution, even asymptotically. Protassov et al. (2002) propose a Monte-Carlo-based solution to this problem based on the method of posterior predictive p-values (Rubin 1984; Meng 1994). In this article we extend the application of Protassov et al.'s solution to the case when we fit the location of a narrow emission line, a situation that was avoided in Protassov et al. (2002).

\subsection{Outline of the Article}

The remainder of the article is organized into four sections. \$2 reviews Bayesian inference and Monte Carlo methods with an emphasis on multimodal distributions, outlines our computation methods, proposes new summaries of multimodal distributions, and describes exploratory statistical methods in this setting. We introduce illustrative examples in @2, 
but detailed spectral analysis is postponed in order to allow us to focus on our proposed methods. In \$3, a simulation study is performed to investigate the statistical properties of our proposed methods, with some emphasis placed on the potential benefits of model misspecification. \$4 presents the analysis of the high redshift quasar PG1634+706, and how to test for the inclusion of the line in the spectral model. Concluding remarks appear in \$5, An appendix outlines the computational methods we developed specifically for fitting the location of narrow emission lines.

\section{Model-Based Statistical Methods}

\subsection{Likelihood-Based and Bayesian Methods}

Using a Poisson model for the photon counts, the likelihood function of the parameter in the spectral model described in $\$ 1.3$ is given by $L\left(\theta \mid \mathbf{Y}^{\text {obs }}\right) \propto \prod_{l \in \mathcal{L}} \Xi_{l}(\theta)^{Y_{l}^{\text {obs }}} \exp \left[-\Xi_{l}(\theta)\right]$ where $\mathbf{Y}^{\text {obs }}=\left\{Y_{l}^{\text {obs }}, l \in \mathcal{L}\right\}$ denotes the observed photon counts. With likelihood-based methods, the parameter value that maximizes the probability of the observed data is generally chosen as an estimate of $\theta$; this estimate is called the maximum likelihood estimate (MLE). In Bayesian methods, prior knowledge for $\theta$ can be combined with the information in the observed data. A prior distribution can be used to quantify information from other sources or to impose structure on a set of parameters. The prior distribution is combined with the likelihood to form a posterior distribution. The prior distribution is denoted by $p(\theta)$ and the posterior distribution by $p\left(\theta \mid \mathbf{Y}^{\mathrm{obs}}\right)$. Bayesian inferences for $\theta$ are based on the posterior distribution. Using Bayes' theorem, the prior distribution and the likelihood function are combined to form the posterior distribution via

$$
\begin{aligned}
p\left(\theta \mid \mathbf{Y}^{\mathrm{obs}}\right) & =\frac{p(\theta) L\left(\theta \mid \mathbf{Y}^{\mathrm{obs}}\right)}{\int p(\theta) L\left(\theta \mid \mathbf{Y}^{\mathrm{obs}}\right) d \theta} \\
& \propto p(\theta) L\left(\theta \mid \mathbf{Y}^{\mathrm{obs}}\right),
\end{aligned}
$$

where the last proportionality holds because $p\left(\mathbf{Y}^{\mathrm{obs}}\right)=\int p(\theta) L\left(\theta \mid \mathbf{Y}^{\mathrm{obs}}\right) d \theta$ does not depend on $\theta$ and, given the observed data, is considered a constant.

Bayesian statistical inferences are made in terms of probability statements, which are quantified using various numerical summaries of the posterior distribution. To illustrate this, we consider a stylized right-skewed distribution; see Figure 1, This distribution is similar to the posterior distribution of the expected counts due to the emission line $k, \lambda_{k}$, because this parameter is necessarily non-negative.

Although from a Bayesian perspective the posterior distribution of a model parameter 
( a )

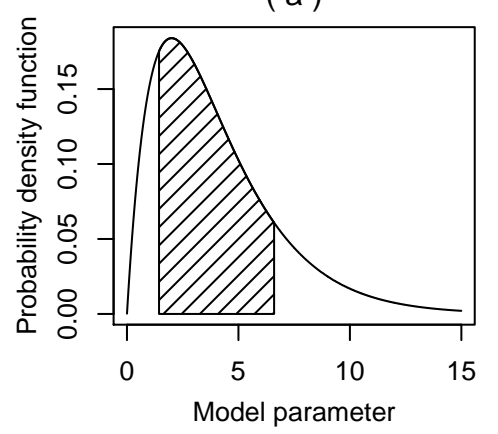

(b)

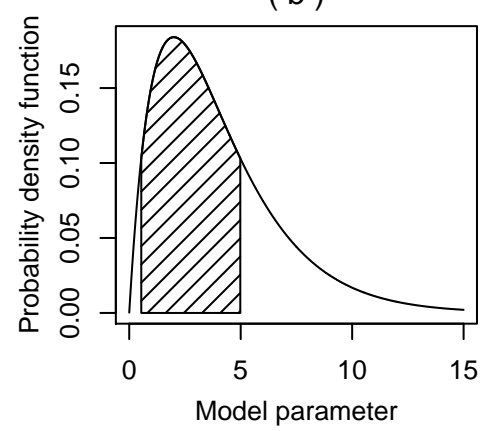

(c)

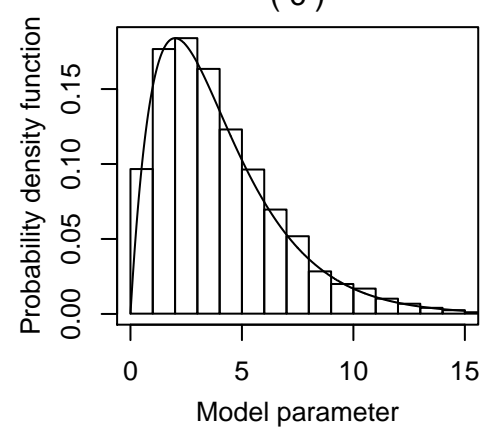

Fig. 1.- Various Summaries of a Right-skewed Distribution. Panels (a) and (b) illustrate the $68 \%$ equal-tail interval and the 68\% HPD interval, respectively. Panel (c) shows that a theoretical probability density function agrees with its Monte Carlo simulation.

is the complete summary of statistical inference for that parameter, it is often useful to summarize the posterior distribution using point estimates or intervals. Commonly used Bayesian point estimates of a parameter are the mean, median, and mode(s) of the posterior distribution. Error bars for the point estimates can be computed based on the variation of the posterior distribution. The equal-tail interval and the Highest Posterior Density (HPD) interval are both commonly used summaries of uncertainty. For example, a $68 \%$ equal-tail posterior interval is the central interval of the posterior distribution and corresponds to the range of values of the parameter above and below which lies exactly $16 \%$ of the posterior probability. A $68 \%$ HPD interval, on the other hand, is the interval of values that contains $68 \%$ of posterior probability and within which the density is never lower than that outside the interval. The $68 \%$ HPD interval is the shortest possible interval that accounts for $68 \%$ of the posterior probability. This is illustrated in Figure 1(a) and (b). The equal-tail interval achieves the same probability as the HPD interval by excluding a more likely region and by including a less likely region. When the posterior distribution is unimodal and symmetric, the equal-tail interval and the HPD interval are identical.

In addition to computing parameter estimates and their error bars, it is often important to check if model assumptions are supported by the data. One way to do this is to generate simulated data under the model and compare the simulated data with the observed data; refer to Protassov et al. (2002) where this strategy is used to determine whether emission line profiles should be included in the spectral model for a gamma-ray burst. If the simulated data vary systematically for the observed data, it is an indication that the model used to simulate the data may not be adequate. In a Bayesian analysis, we might generate such 
simulated data from the posterior predictive distribution, denoted by $p\left(\widetilde{\mathbf{Y}} \mid \mathbf{Y}^{\text {obs }}\right)$, i.e.,

$$
\begin{aligned}
p\left(\tilde{\mathbf{Y}} \mid \mathbf{Y}^{\mathrm{obs}}\right) & =\int p\left(\tilde{\mathbf{Y}} \mid \mathbf{Y}^{\mathrm{obs}}, \theta\right) p\left(\theta \mid \mathbf{Y}^{\mathrm{obs}}\right) d \theta \\
& =\int L(\theta \mid \tilde{\mathbf{Y}}) p\left(\theta \mid \mathbf{Y}^{\mathrm{obs}}\right) d \theta
\end{aligned}
$$

where $\tilde{\mathbf{Y}}$ represents an unknown future observation and the last equation follows because $\tilde{\mathbf{Y}}$ and $\mathbf{Y}^{\text {obs }}$ are conditionally independent given $\theta$. In words, the posterior predictive distribution averages the likelihood function over the posterior distribution of $\theta$. Data are simulated from the posterior predictive distribution and then used to make predictive inferences; see $\$ 4.3$ for details.

Posterior simulation plays a central part in applied Bayesian analysis because of the usefulness of a simulation that can often be relatively easily generated from a posterior distribution. In performing simulations, given a large enough sample, a histogram of a Monte Carlo simulation can provide practically complete information about an actual posterior distribution. Figure 1(c) shows that the histogram of the Monte Carlo simulation carries the same information as the posterior distribution itself. Thus, once a Monte Carlo simulation is obtained, it can be used to compute the mean, variance, percentiles, and other summaries of the posterior distribution. In particular, with a random simulation of size $N$, the posterior mean can be approximated as

$$
\mathrm{E}\left(\theta \mid \mathbf{Y}^{\mathrm{obs}}\right)=\int \theta p\left(\theta \mid \mathbf{Y}^{\mathrm{obs}}\right) d \theta \approx \frac{1}{N} \sum_{\ell=1}^{N} \theta^{(\ell)},
$$

where $\left\{\theta^{(\ell)}, \ell=1, \ldots, N\right\}$ is a simulation from the posterior distribution, $p\left(\theta \mid \mathbf{Y}^{\text {obs }}\right)$. A $68 \%$ equal-tail posterior interval is computed by generating a Monte Carlo simulation of size $N$ from the posterior distribution, sorting the simulated values into increasing order, and choosing the $[0.16 N]$ th and the $[0.84 N]$ th values in the list. With the Monte Carlo simulation, a $68 \%$ HPD region is computed by segmenting the range of possible parameter values into bins, approximating the posterior probability of each bin as the proportion of the simulated values in that bin, and computing a region by beginning with the bin with the largest posterior probability and adding additional bins in the order of their posterior probabilities until the resulting region contains at least $68 \%$ of the posterior probability.

\subsection{Outline of Computational Strategies}

Our search for a narrow emission line begins by finding modes of its posterior distribution that correspond to plausible locations of an emission line. To find the modes (and to compte 

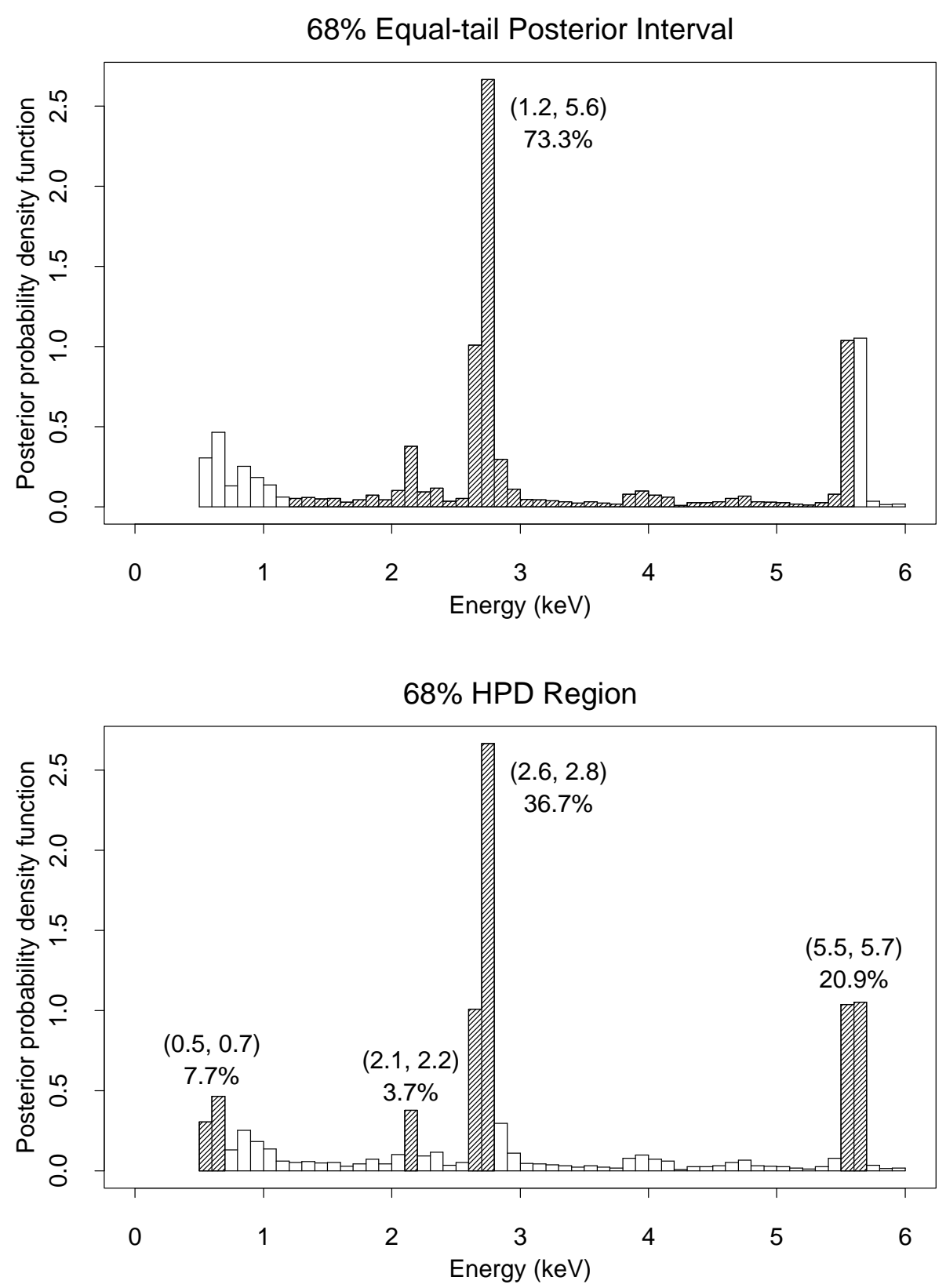

Fig. 2.- Comparison of an Equal-tail Interval and an HPD Region Computed with a Monte Carlo Simulation. The shaded interval in the top panel indicates a $68 \%$ equal-tail interval and the shaded region in the bottom panel indicates a $68 \%$ HPD region. On the top of each shaded area, its range of energies and the corresponding posterior probability accounted for are shown. The HPD region is not an interval, and is much more informative as to the likely values of the line location. Here, we use a coarse binning for illustrative purposes. In our actual analysis, we use finer binning to construct more precise equal-tail intervals and HPD regions. The shape of the histogram is typical of what we observe for the location of a relatively weak emission line. 
the profile posterior distribution), we use an algorithm optimized for this problem (i.e., the Rotation(9) EM-type algorithm, see Appendix A and van Dyk \& Park (2004) for details). Because the posterior distribution of the line location is highly multimodal (see \$2.3), the algorithm is run using multiple starting values selected across the entire energy range of possible line locations, e.g., 50 starting values for the line location equally spaced between $1.0 \mathrm{keV}$ and $6.0 \mathrm{keV}$. Using multiple starting values enables us to identify the important local modes of the posterior distribution. It is important to use enough starting values to ensure all of the important modes are identified. This is a standard strategy, long advocated in texts on Bayesian data analysis (Gelman et al. 1995, 2003) and is closely related to the computation of the profile posterior distribution described in \$2.4. The profile posterior distribution is computed by fixing the line location at each value of a fine grid, finding the posterior modes of the other model parameters, and plotting the resulting maximum posterior probability as a function of the line location. This procedure corresponds to the projected delta-chi-square method in the chi-square setting, see Lampton et al. (1976) and Press et al. (1992). Mode finding also begins with a fine grid of starting values, but we run the mode finder allowing all parameters including the line location to be fit.

After the modes are found, Monte Carlo simulation techniques optimized to this problem can be run to further investigate the uncertainty of the possible line locations. We employ state-of-the-art MCMC samplers, i.e., Partially Collapsed Gibbs (PCG) I for the delta function emission line and PCG II for the Gaussian emission line to obtain the posterior distribution of line location; see Appendix A, van Dyk \& Park (2008), and Park \& van Dyk (2008) for details. To ensure the convergence of a Markov Chain constructed by the MCMC samplers, we run multiple chains with overdispersed starting values (e.g., $1 \mathrm{keV}, 3 \mathrm{keV}$, and $5 \mathrm{keV}$ for the line location parameter) and monitor the convergence qualitatively and quantitatively. For example, we compute the estimate of the potential scale reduction (Gelman \& Rubin 1992), denoted by $\widehat{R}^{1 / 2}$, for all parameters of interest. If $\widehat{R}^{1 / 2}$ is near 1 (e.g., below 1.2) for each of the parameters, we collect the second halves of the chains together and use these Monte Carlo draws for inference; see Gelman \& Rubin (1992) for theoretical justification and discussion. There are of course many strategies that one might employ to construct efficient Monte Carlo samplers. Methods based on annealing or tempering, or that use explicitly parallel methods are often useful for exploring multimodal posterior distributions. Given the low autocorrelation of the simulated values produced by our method, we have not pursued these strategies. 


\subsection{Summarizing Multimodal Posterior Distributions}

Fitting a narrow emission line often tends to yield a highly multimodal posterior distribution of the line location, as shown in Figure 2. Thus, we are interested in the two types of intervals for the posterior distributions: an equal-tail posterior interval and an HPD region. Figure 2 illustrates a $68 \%$ equal-tail posterior interval and a $68 \%$ HPD region for the line location when its posterior distribution is highly multimodal. This is only an example of a highly multimodal posterior distribution. We come to the details of our analysis of a line location in 93 , The $68 \%$ equal-tail posterior interval is a central interval, so that it includes segments with nearly zero posterior probability here and there within the interval. Because the posterior distribution of the line location in Figure 2 is multimodal, the $68 \%$ HPD region consists of the four shaded disjoint intervals; notice that the height of each of the histogram bars outside the HPD region is less than that of those within the region. In such a multimodal posterior distribution, the HPD region not only is shorter in length but also conveys more information as to likely locations of the line than does the equal-tail interval.

In Figure 2, we use a coarse binning to illustrate the distinction between the equaltail posterior interval and HPD region. This results in a posterior region that is relatively imprecise; in our spectral analysis, we use a finer binning to construct a more precise region from a Monte Carlo simulation. When the posterior distribution of the line location is plotted with the same fine binning as the Chandra energy spectrum, however, it may not be smooth due to Monte Carlo errors, as shown in the top panel of Figure 3. An HPD region computed from the unsmoothed posterior distribution may result in a combination of too many posterior intervals. To avoid such fragmentation of HPD regions, we use Gaussian kernel smoothing to smooth the posterior distribution before we compute HPD regions. The middle panel of Figure 3 presents the smoothed posterior distribution resulting from applying Gaussian kernel smoothing with standard deviation equal to the bin size of the Chandra energy spectrum, i.e., $0.01 \mathrm{keV}$. The smoothed posterior distribution smooths out lower posterior probabilities but does not flatten higher posterior probabilities too much.

We propose a new graphical summary to better describe the HPD regions of a (smoothed) multimodal posterior distribution. An HPD graph is constructed by plotting a series of HPD regions against their corresponding HPD levels. For example, for the data set used to compute the posterior distribution in Figure 3, we compute 100 HPD regions, one for each of 100 levels, $1 \%, 2 \%, \ldots$, and $100 \%$. Each of these regions is a union of possible values of the line location. We can plot the line location on the horizontal axis and the level on the vertical axis. Each of the $100 \mathrm{HPD}$ regions can then be plotted as a union of horizonal line intervals at the appropriate level of the HPD region on the vertical axis. The resulting HPD graph lets us visualize many HPD regions with varying levels, so that all the important 

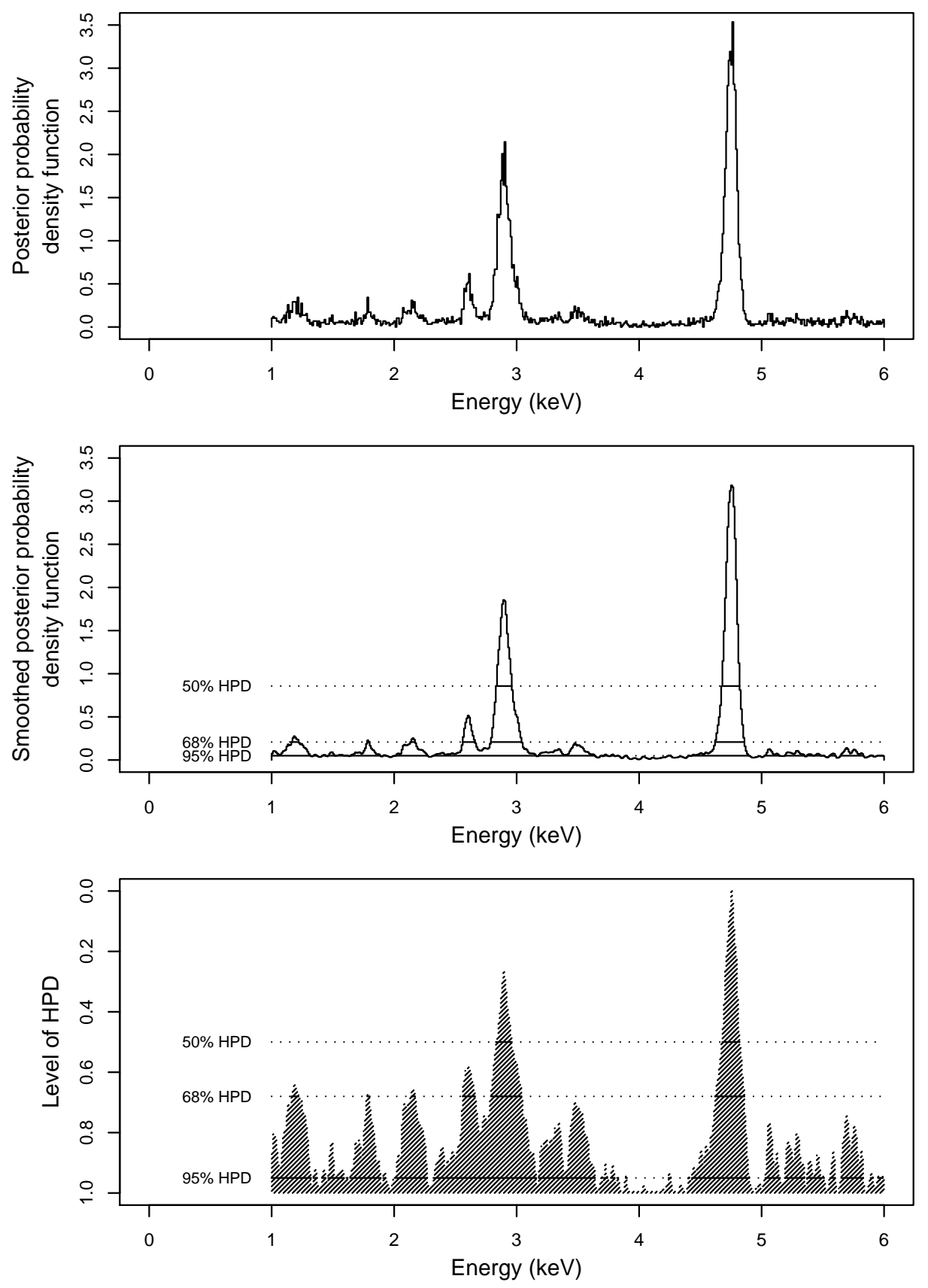

Fig. 3.- Comparing an Unsmoothed Marginal Posterior Distribution with its HPD Graph computed with Gaussian Kernel Smoothing. The top panel presents an unsmoothed posterior distribution computed from Monte Carlo draws, plotted with the same resolution as the Chandra energy spectrum. In the middle panel, the posterior distribution is smoothed using Gaussian kernel smoothing with standard deviation $0.01 \mathrm{keV}$, the width of one energy bin in the Chandra spectrum. Based on the smoothed posterior distribution, we compute 100 HPD regions with levels ranging from $1 \%$ to $100 \%$. These intervals are plotted against their levels in the HPD graph shown in the bottom panel. The horizontal solid lines in the middle and bottom panels are the 50\%,68\%, and 95\% HPD regions, and the horizontal dotted lines are the intervals outside each HPD region. 
modes of a multimodal distribution can be effectively summarized with their relative posterior probabilities. As an illustration, we computed the three HPD regions with levels 50\%, $68 \%$, and $95 \%$, and plotted them in the middle panel of Figure 3 along with the smoothed posterior distribution. The solid lines indicate the disjoint intervals that compose each HPD region, and the dotted lines the intervals outside the HPD region. The bottom panel in Figure 3 shows the HPD graph in grey with the three HPD intervals from the middle plot superimposed.

When there are two parameters of interest, the 1-D HPD graph can be extended to a 2-D HPD graph. That is, a joint posterior distribution is computed from a Monte Carlo simulation by using bivariate Gaussian kernel smoothing and used to construct 2-D HPD regions with various levels. These HPD regions can then be plotted with different shades of grey. For example, Figure 4 shows the 2-D HPD graph computed for the joint posterior distribution of two possible line locations; see $\$ 3.2$ for further discussion on the scatter plot. The left panel of Figure 4 is an unsmoothed joint posterior distribution. After applying bivariate Gaussian kernel smoothing, 10 HPD regions (one for each of 10 levels, 10\%, 20\%, $\ldots$, and $100 \%$ ) are computed and plotted with different shades of grey from black (10\%) to white $(100 \%)$, as shown in the right panel of Figure 4. HPD regions with lower levels contain pixels with higher posterior probabilities, so that darker pixels indicate more probable regions of the two line locations.

Multimodal likelihoods and posterior distributions pose another challenge for statistical analysis. The calibration of many standard statistical procedures is based on the asymptotic Gaussian nature of the likelihood and posterior distribution. Thus, standard methods for computing error bars and confidence intervals rely on the likelihood being at least approximately Gaussian. When the likelihood function exhibits the multimodal features that we see in Figures 3 and 4 , these standard results simply do not apply. One consequence of this is that the nominal level of an interval may not match its frequency coverage. That is, if we were able to repeat our observation many times, we would find that percentage of intervals that contain the true line location might differ from the nominal level of the interval. In \$3, we find that the posterior distribution is highly multimodal when the true emission line is weak or there are several emission lines in a spectrum. In this case, we also find that by misspecifying the model we are able to improve the frequency properties of our proposed method. In particular, we find that using a delta function line profile improves the properties of our procedure even when the true line has appreciable width. 

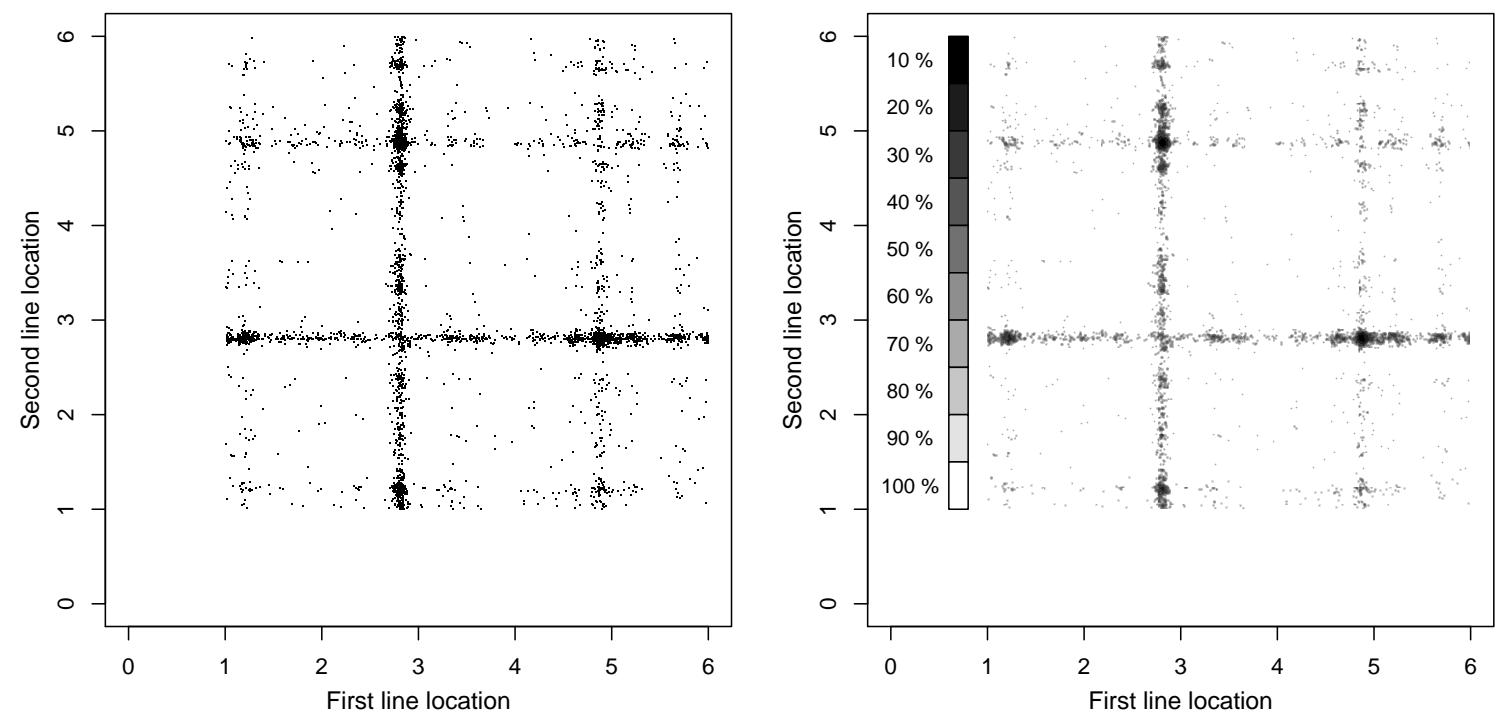

Fig. 4.- Comparing an Unsmoothed Joint Posterior Distribution with its 2-D HPD Graph computed with Bivariate Gaussian Kernel Smoothing. The left panel presents an unsmoothed joint posterior distribution computed from Monte Carlo draws, plotted with the same resolution as the Chandra energy spectrum. The unsmoothed posterior distribution is smoothed using bivariate Gaussian kernel smoothing with covariance matrix $\left(\begin{array}{cc}0.01^{2} & 0 \\ 0 & 0.01^{2}\end{array}\right)$, where each marginal standard deviation corresponds to the size of an energy bin in the Chandra spectrum. Based on the smoothed posterior distribution, we compute ten 2-D HPD regions with levels ranging from $10 \%$ to $100 \%$. These regions are plotted with different shades of grey in the 2-D HPD graph shown in the right panel. 


\subsection{Exploratory Data Analysis}

As discussed in $₫ 2.3$, the posterior distribution of a narrow emission line location tends to be highly multimodal. In this case, the profile posterior distribution can be used as a handy and quick-to-compute summary of the posterior distribution to explore the possible locations of a spectral line. The profile posterior distribution is the posterior distribution of a parameter evaluated at the values of the other parameters that maximize the posterior distribution. The profile posterior distribution is a Bayesian analogue to the profile likelihood, a standard likelihood method for dealing with nuisance parameters; see Venzon \& Moolgavkar (1988) or Critchley et al. (1988) for applications of the profile likelihood. In the context of parameter

estimation, the distribution of the minimum $\chi^{2}$ statistic described by Lampton et al. (1976) is closely related to the profile posterior distribution.

Generally we do not advocate using the profile posterior distribution as a substitute for the marginal posterior distribution because interval or region estimates computed with the profile posterior distribution have rather unpredictable statistical properties. The marginal posterior distribution is obtained by integrating out (i.e., averaging over) the other parameters and computing the marginal posterior distribution requires sophisticated numerical integration methods such as MCMC especially when the dimension of the nuisance parameters is large. However, the profile posterior distribution can be computed without MCMC and, as an analogue to the marginal posterior distribution, can be used to roughly examine the posterior distribution of a model parameter. Thus, we believe the profile posterior distribution is well suited for the initial exploration of the data because it gives a clear and reliable set of potential locations for spectral lines.

The profile posterior distribution can be computed on a fine grid of the possible values of the line location by running an optimizer to maximize over the other model parameters for each value of the line location on the grid; we recommend using stable optimizers such as EM-type algorithms (van Dyk \& Park 2004). The profile posterior distribution of the line location computed in this way is computationally less demanding and cheaper in terms of CPU time than the marginal posterior distribution produced by Monte Carlo methods such as MCMC.

\section{Simulation Study}

Our simulation study is conducted to assess the validity of the highly structured multilevel spectral model discussed in $\$ 1.3$, to illustrate the connection between the multimodal posterior distribution of a single narrow emission line and evidence for multiple lines, to 
illustrate the possible advantage of the misspecification of an emission line width, and to illustrate the relationship among Gaussian line parameters.

We consider the following six cases that we believe are representative of the cases that are of general interest:

Case 1 : There is no emission line in the spectrum.

CASe 2 : There is a narrow Gaussian emission line at $2.85 \mathrm{keV}$ with $\mathrm{SD} b=0.04 \mathrm{keV}$ and equivalent width, $\mathrm{EW}=0.198 \mathrm{keV}$ in the spectrum.

CAse 3 : There is a moderate Gaussian emission line at $2.85 \mathrm{keV}$ with $\mathrm{SD}=0.21 \mathrm{keV}$ and $\mathrm{EW}=0.661 \mathrm{keV}$ in the spectrum.

CASE 4 : There is a narrow Gaussian emission line at $2.85 \mathrm{keV}$ with $\mathrm{SD}=0.04 \mathrm{keV}$ and $\mathrm{EW}=0.659 \mathrm{keV}$ in the spectrum.

CAse 5 : There are two narrow Gaussian emission lines with $\mathrm{SD}=0.04 \mathrm{keV}$, one at $1.20 \mathrm{keV}$ with $\mathrm{EW}=0.139 \mathrm{keV}$ and the other at $2.85 \mathrm{keV}$ with $\mathrm{EW}=0.198 \mathrm{keV}$ in the spectrum.

CAse 6 : There are two narrow Gaussian emission lines with $\mathrm{SD}=0.04 \mathrm{keV}$, one at $1.20 \mathrm{keV}$ with $\mathrm{EW}=0.139 \mathrm{keV}$ and the other at $2.85 \mathrm{keV}$ with $\mathrm{EW}=0.659 \mathrm{keV}$ in the spectrum.

That is, each spectrum has either 0, 1, or 2 lines. Typical Chandra data are recorded in an energy grid with bin width $0.01 \mathrm{keV}$. Thus, the narrow emission line (i.e., CAses 2, 4, 5, and 6) corresponds to about 17 energy bins and the moderate one (i.e., CASE 3) about 85 energy bins, using \pm 2 standard deviations. As compared to the Chandra resolution, the delta function emission line profile corresponds to 1 energy bin and thus it does not correctly specify the width of the Gaussian emission lines in this simulation. Through the simulation study, however, we illustrate possible advantage of this model misspecification in producing valid and efficient estimates and associated uncertainties for the line location. We show that using delta functions emission lines in the model is a useful strategy even when the true line occupies multiple bins.

For each of the six spectra, we generate twenty test data sets (120 data sets in total) each with about 1500 counts similar to the observed number of counts in the Chandra X-ray spectrum of PG1634+706 analyzed in $₫ 4$, mimicking the real data situation. Each spectrum

\footnotetext{
${ }^{\mathrm{b}} \mathrm{SD}$ stands for standard deviation.
}

${ }^{\mathrm{c}}$ The equivalent width is defined as $\mathrm{EW}=\lambda /\left[f\left(\theta^{C}, \mu\right) \cdot \mathrm{ARF} \cdot\right.$ Exposure time $]$. 
has a power law continuum with $\alpha^{C}=3.728 e-5$ and $\beta^{C}=1.8$. Our simulation is done with Sherpa software (Freeman et al. 2001) in CIAd), assuming the Chandra responses (effective area and instrument response function) and no background contamination.

\subsection{Validity of Delta Function Line Profiles}

For each of the test data sets, we run state-of-the-art MCMC samplers to fit a spectral model with a single delta function emission line. Based on the Monte Carlo draws collected from the multiple chains of the MCMC samplers, the top two rows of Figure 5 present the marginal posterior distribution of the delta function line location for one simulation under each of the six cases; the vertical dashed lines represent the true line locations. The marginal posterior density is smoothed using Gaussian kernel smoothing with standard deviation $0.01 \mathrm{keV}$, as described in $\$ 2.3$.

When there is no emission line in the spectrum (i.e., CASE 1), the posterior distribution of the delta function line location is highly multimodal. In the case of a weak narrow Gaussian emission (i.e., CASE 2), the marginal posterior distribution often remains highly multimodal, but one mode typically identifies the true line location. In practice, the local mode(s) of such a highly multimodal posterior distribution may suggest plausible line locations and show evidence for multiple lines; see §3.2. Even with a moderate line (i.e., CASE 3), the true line location appears well estimated with the marginal posterior distribution of the delta function line location. As we shall see in Table 1, however, in this case the resulting posterior region under-covers the true values because the true line is 85 times wider than the specified model. With the strong narrow Gaussian line (i.e., CAsE 4), the posterior distribution of the line location tends to be unimodal, and the posterior mode correctly identifies the true line location. The posterior distribution for CASE 5 in Figure 5 is bimodal, with the modes corresponding to the two true line locations. When multiple lines are present in a spectrum, the posterior distribution of the single line location can be multimodal, as shown in the CASE 5 of Figure 5. Thus the multiple modes may be indicative of multiple lines; see $\$ 3.2$ for details. When one of two narrow Gaussian emission lines is much stronger (i.e., Case 6), the single delta function line model tends to identify only one of the two true line locations. To visualize the uncertainty of the fitted delta function line location(s), the bottom two rows of Figure 5 show the HPD graphs constructed with 100 HPD regions as described in 92.3 .

\footnotetext{
${ }^{\mathrm{d}}$ The software is publicly available on http://cxc.harvard.edu/ciao.
} 

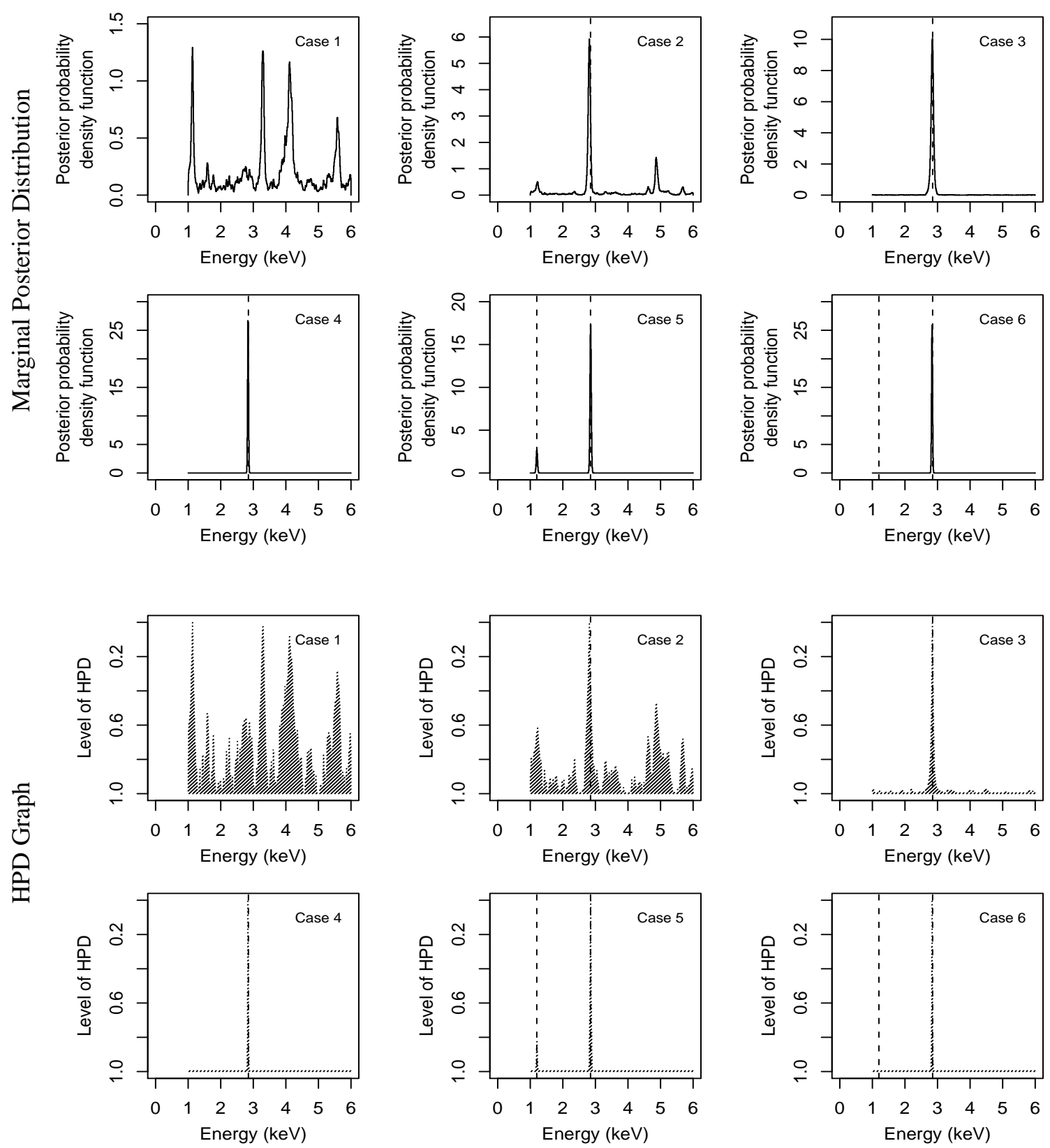

Fig. 5.- The Marginal Posterior Distribution and HPD Graph of the Line Location for One Simulation Under Each of the Six Cases in the Simulation Study. There is no emission line in the spectrum of CASE 1; the spectra of CASES 2, 3, and 4 include one narrow or moderate emission line; and the spectra of CASEs 5 and 6 include two narrow emission lines. The top two rows illustrate the smoothed marginal posterior distributions of the line location for the six spectra in the simulation study, computed using Gaussian kernel smoothing with standard deviation $0.01 \mathrm{keV}$. The corresponding HPD graphs constructed with 100 HPD regions are presented in the bottom two rows; refer to Figure 3. The vertical dashed lines represent the locations of the true emission lines. 


\subsection{Connection Between Multimodality and Multiple Lines}

The multimodality in the marginal posterior distribution of a single line location may indicate the existence of multiple lines in a spectrum, provided the lines are well separated. When a model is fitted with one emission line, modes in the likelihood function of the line location correspond to ranges of energy with excess emission relative to the continuum. Multiple modes in the likelihood indicate that there are multiple ranges of energy with such excess emission. The height of the mode is indicative of the degree of excess. Thus, if there are several emission lines, we might expect to see several corresponding modes in the likelihood. If there is one energy range that dominates in terms of excess emission, however, it corresponds to the dominate mode of the likelihood. Thus, if there are lines of very different intensities, only the strongest ones may show up as a mode of the likelihood. This can be seen by comparing CaSe 5 and CASE 6 in Figure 5 .

If there is evidence for multiple lines in a spectrum or if we suspect multiple lines a priori, we can fit a model with two or more lines. We illustrate this using simulated data under Case 2 (one narrow line) and CASE 5 (two narrow lines). Beginning with Case 2, the actual spectrum has only one line, but we investigate what happens when we fit two lines to this data. A scatterplot of the two fitted line locations identified when fitting two emission lines to one of the data sets generated under CASE 2 is presented in the top left panel of Figure 6. There is a label switching problem between the two fitted line locations because of the symmetry of the emission lines in the model. We can remove the symmetry by imposing a constraint on line locations. To do this, we first fit the model with a single delta function line profile and compute the posterior mode of its line location. Returning to the model with two fitted delta functions, we separate the two fitted line locations by setting the "first" line location to be the one closest to the posterior mode. In Case 2, the posterior mode for the single line location is $2.815 \mathrm{keV}$, so that the first line location is the line location closest to $2.815 \mathrm{keV}$ and the second line is the other location. The two panels in the top right corner of Figure 6] show the resulting marginal posterior distributions of the two fitted line locations. As shown in the figure, the marginal posterior distribution of the first line location correctly identifies the true line location. The spectrum used to generate the data under CASE 2 has no second line, so that the marginal posterior distribution of the second line is highly multimodal. In practice, we may take the local mode(s) in the second marginal posterior distribution as candidates for another line location. However, the resulting HPD regions for the second line are wide, indicating that either there is no second line or if there is, it cannot be well identified.

When two emission lines are present in a spectrum, we follow the same procedure as

illustrated using the data generated under CASE 5. The bottom left panel of Figure 6 shows 

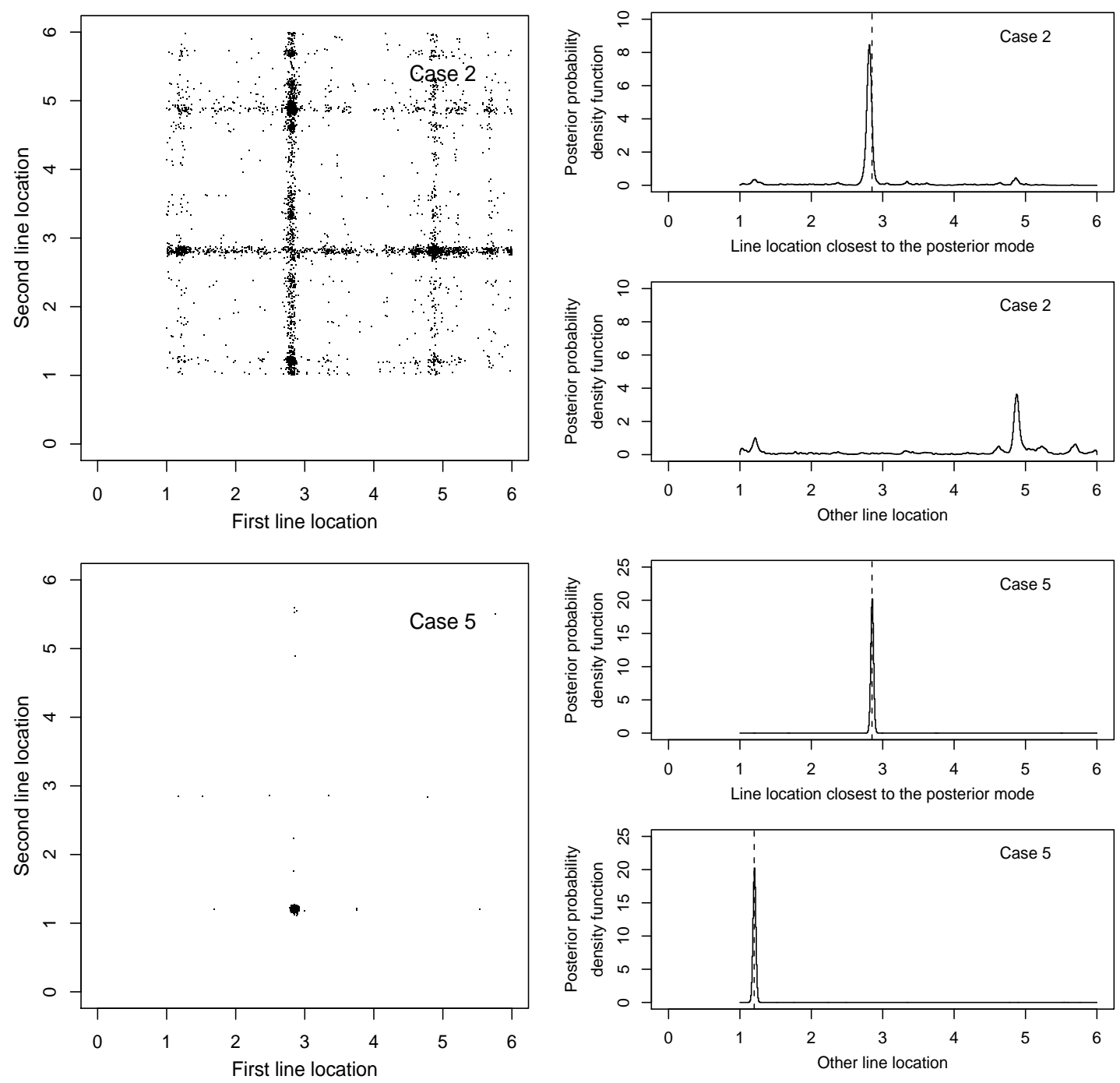

Fig. 6.- The Joint Posterior Distribution and the Corresponding Marginal Posterior Distributions of Two Line Locations in CASE 2 (one narrow emission line) and CASE 5 (two narrow emission lines). The vertical dashed lines in the right panels represent the locations of true emission lines. 
the scatterplot of two line locations identified in the spectrum of CASE 5. Label-switching is handled as above using the posterior mode for the single line location, which is computed as $2.855 \mathrm{keV}$. The fitted marginal posterior distributions of the first and second line locations are given in the bottom right corner of Figure 6. When there are two emission lines in a spectrum, the two true line locations are precisely specified by the two marginal posterior distributions. This is a verification of what is suggested by the multiple modes in the marginal posterior distribution of the single line location shown in Figure 5 .

\subsection{Possible Advantage of Model Misspecification}

The possibility of model misspecification when using a delta function to model an emission line depends on both the width of the true line and the resolution of the detector. Misspecification only occurs when the line is not contained in one energy bin, and we shall illustrate that such misspecification only has statistical consequences for the fitted line location if it is very severe. Indeed there can be a possible statistical advantage of using a delta function rather than a Gaussian line if we know the spectral line is not too wide. As a toy example, consider a simple Gaussian model with known standard deviation: When $Y \sim \mathrm{N}(\mu, \sigma)$, a $95 \%$ confidence interval for $\mu$ is given by $Y \pm 1.96 \sigma$. If we misspecify the modal as $Y \sim \mathrm{N}(\mu, \varsigma)$ with $\varsigma<\sigma$, the resulting interval for $\mu$ is shorter and has lower coverage. We similarly underrepresent the error bars of an emission line location when we use a delta function for a line that is not contained in one energy bin. We expect this to reduce both the length and coverage of the confidence regions. The advantage or disadvantage of this strategy is not immediately clear, however, since the nominal coverage of the intervals is based on an asymptotic Gaussian approximation to the posterior distribution which clearly does not apply in this setting. Nonetheless, our simulation study illustrates that the use of a delta function line profile can result in a shorter and more informative HPD region while maintaining good coverage.

We now turn to the computation of HPD regions and the possible statistical advantage of using delta functions in place of narrow Gaussian emission lines. We fit a spectral model that includes a single delta function line or a single narrow Gaussian line to the twenty simulated data sets generated under each of the 6 cases. After smoothing the marginal posterior distribution using Gaussian kernel smoothing, we construct 95\% HPD regions for the line location, as shown in Figure 7. For visual clarity, we present results only for the first ten simulated data sets in Figure 7, results from all twenty simulated data sets are discussed in Table 1. Because there is no emission line in the spectrum used to simulate data under CASE 1, the 95\% HPD regions for the line location are very wide and show large 

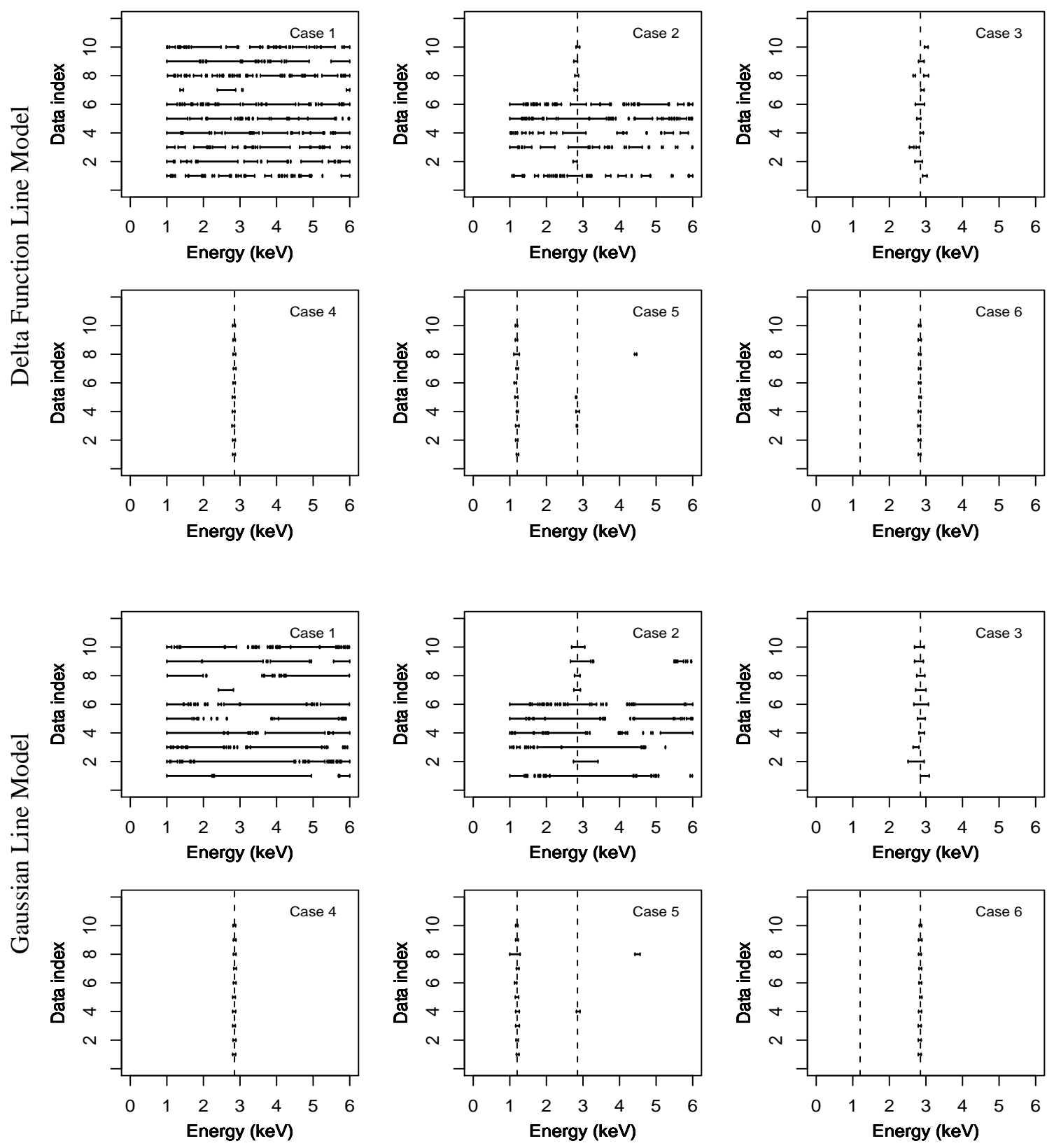

Fig. 7.- 95\% HPD Regions for the Delta Function Line Location and Gaussian Line Location in the Simulation Study. The HPD regions are all computed under a model containing a single emission line and plotted against the index of the first ten simulated data sets. The intervals produced when using a delta function line profile tend to be somewhat narrower and more precise. The vertical dashed lines represent the locations of true emission lines. 
Table 1: Summary of 95\% HPD Regions for the Line Location in the Simulation Study.

\begin{tabular}{|c|c|c|c|c|c|}
\hline \multirow[b]{2}{*}{ Case } & \multirow[b]{2}{*}{ Line type $\mathrm{e}^{\mathrm{e}}$} & \multicolumn{2}{|c|}{ Delta function line } & \multicolumn{2}{|c|}{ Gaussian line } \\
\hline & & Coverage $^{f}$ & Mean length & Coverage $^{f}$ & Mean length \\
\hline 1 & no lines & $\mathrm{NA}$ & 3.354 & NA & 3.613 \\
\hline 2 & one narrow line & $85 \%$ & 0.722 & $100 \%$ & 1.489 \\
\hline 3 & one moderate line & $65 \%$ & 0.164 & $95 \%$ & 0.263 \\
\hline 4 & one narrow line & $100 \%$ & 0.068 & $100 \%$ & 0.075 \\
\hline 5 & two narrow lines & $95 \%$ & 0.081 & $95 \%$ & 0.137 \\
\hline 6 & two narrow lines & $100 \%$ & 0.068 & $100 \%$ & 0.076 \\
\hline total & narrow line $(\mathrm{s})$ & $95 \%$ & 0.235 & $98.8 \%$ & 0.444 \\
\hline
\end{tabular}

uncertainties for the fitted line location. When there is at least one strong emission line (i.e., CASES 3, 4, and 6), both line models produce comparable HPD regions, although those computed under the Gaussian line model appear somewhat wider. The tradeoff between the two line models becomes evident when there is no strong emission line in the spectrum (i.e., Cases 2 and 5). In Case 2, the 95\% HPD regions for a single Gaussian line location are somewhat wider. With the same nominal level, the delta function line model yields more compact and informative HPD regions. An added advantage of the delta function line model occurs in CASE 5 when the $95 \%$ HPD regions for a single delta function line location consist of two disjoint HPD intervals which simultaneously contain the two true line locations; this behavior is more often observed with the delta function line model (2 times out of 20) than the Gaussian line model (1 time out of 20 ).

To more closely inspect the advantage of model misspecification, we evaluate the coverage of the true line locations and the mean length of the $95 \%$ HPD regions. For each of the 6 spectra, Table 1 shows the percentage of the twenty $95 \%$ HPD regions containing at least one true line location along with the mean length of the regions. In CASE 1, there is no emission line in the spectrum and there should therefore be great uncertainties about the line location. Thus both line models produce comparably wide HPD regions for the line location on average. When a spectrum has at least one emission line, the delta function line model yields HPD regions of smaller mean length and with better coverage rates. The one

\footnotetext{
eThe narrow emission lines are 17 bins wide (four standard deviations, i.e., $0.17 \mathrm{keV}$ ); moderate emission lines are 85 bins wide (i.e., $0.85 \mathrm{keV}$ ).

${ }^{\mathrm{f}}$ The coverage is the percentage of twenty $95 \%$ HPD regions containing at least one true line location.
} 
exception is CASE 3 that includes one moderate emission line whose location is significantly undercovered with the delta function line model. In this case, the 85-bin (i.e., $0.85 \mathrm{keV}$ ) wide line is very broad, as compared to the delta function line profile which corresponds to one energy bin (i.e., $0.01 \mathrm{keV}$ ). Although the delta function appears to be efficient in identifying lines this wide, the model underrepresents the uncertainty in their location. When all emission lines are narrow (i.e., CASEs 2, 4, 5, and 6), the misspecification of the line width seems to show an advantage. The last row of Table 1 summarizes the 95\% HPD regions when there is at least one narrow emission line in the spectrum, i.e., an emission line with a width of 17 bins (i.e., $0.17 \mathrm{keV}$ ). The delta function line model produces HPD regions with $53 \%$ the mean length of HPD regions resulting from the Gaussian line model, with coverage closer to the nominal $95 \%$ rate.

Because fitting a spectral model involves MCMC sampling that is computationally expensive and requires some supervision, exhaustive simulations are difficult to carry out. In addition, the results may depend on the line location, line strength, line width, characteristics of the continuum, sample size, and so on. Based on the simulation study, however, we conclude that the delta function line model is useful for exploratory data analysis and for inference when the true line is believed to be narrow. From a computational point of view, an additional advantage is a significant reduction in the computational time when using a delta function, owing largely to the fact that the line width need not be fit. While the delta function line model enjoys the advantages of model misspecification when searching for the locations of narrow emission lines, it is not designed to estimate the other line parameters. The delta function emission line model underestimates the width, intensity, and EW of the emission line. When making inference for these line parameters, the delta function emission line model should not be used unless an emission line is truly narrow relative to the resolution of the detector.

\subsection{Summary of Line Parameters}

When fitting a Gaussian emission line profile, the fitted line intensity may be correlated with the fitted line width. To examine the relationship among the Gaussian line parameters, Figure 8 shows the pairwise joint posterior distributions of the Gaussian line location, the line $\mathrm{SD}$, and the log of the line intensity. The two rows in Figure 8 correspond to the results from two simulated data sets, where the posterior distribution of the line location tends to be unimodal or (highly) multimodal. The two simulated data sets are both from CAsE 2 but encompass the two typical types of posterior distributions we see in CASES 2, 3, 4, 5, and 6 .

As the possible location of a Gaussian emission line is shifted away from its posterior 

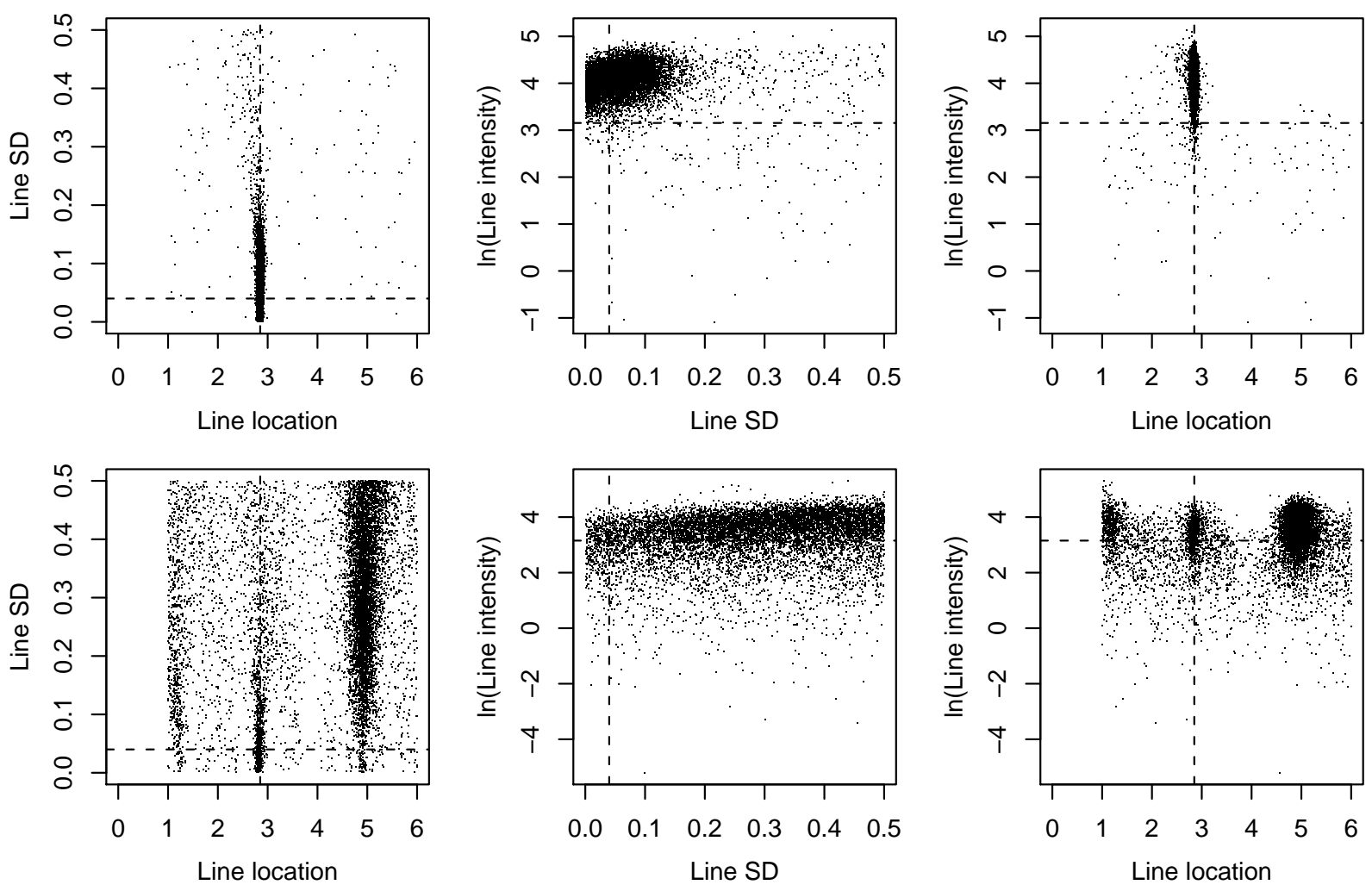

Fig. 8.- Joint Posterior Distributions of the Gaussian Line Location, the Line SD, and the Log of the Line Intensity from the Simulation Study. The two rows correspond to two typical types of test data in CASE 2, where the first row represents the test data with the line location having a unimodal posterior distribution and the second row the test data with the line location having a multimodal posterior distribution. The dashed lines represent either the true location $(2.85 \mathrm{keV})$, the true standard deviation $(0.04 \mathrm{keV})$, or the true log intensity (3.15 photons) of a narrow and weak Gaussian emission line in Case 2 of our simulation study. 
mode(s), the line width tends to increase in order for the Gaussian emission line to be wide enough to encompass the excess emission. When the line location is not well specified, fitted values far from the true line location may be consistent with the data so long as the width and equivalent width of the line are sufficiently large. Thus, the joint posterior distribution of the line location and line width tends to have $\mathrm{V}$-shape at each mode of the line location. This behavior is illustrated in the left panels of Figure 8. A larger fitted value of the line width, in turn, increases the Gaussian line intensity, resulting in an overall positive relationship in the middle panels of Figure 8 . As a result, the line intensity tends to increase as the line location moves away from each of its modes, as illustrated in the right panels of Figure 8 .

\section{Analysis of the Quasar PG1634+706}

\subsection{The High Redshift Quasar PG1634+706}

X-ray spectra of many sources are available in Chandra archivesg. We apply our methods to the calibration source, PG1634+706 that was observed 6 times during the first year of the Chandra mission. Only one observation (obs-id 1269) has been analyzed and published (Haro-Corzo et al. 2007) and it indicates that a narrow iron line was detected in this source. We include all available data sets in our analysis to evaluate the location and significance of the line. We focus on a single narrow emission line that might correspond to either the narrow component or one of the two peaks of the broad component, of the Fe-K-alpha line discussed in \$1.1. In principle an analysis might include two or three delta functions in an effort to simultaneously identify all three features. As we shall discuss, however, we find only marginal evidence for one feature, and thus did not pursue the simultaneous fitting of multiple features.

PG1634+706 (redshift $z=1.334$ ) is a radio quiet and optically bright quasar (Steidel \& Sargent 1991). It is very luminous in X-rays with the $2-10 \mathrm{keV}$ band luminosity exceeding $10^{46} \mathrm{erg} \mathrm{s}^{-1}$ (Jiménez-Bailón et al. 2005). The iron emission line in such luminous sources is expected to be weaker than in lower luminosity AGN (Nandra et al. 1997). The quasar was observed with ASCA (George et al. 2000) and XMM-Newton (Page et al. 2005) and no line was detected at the energy of the $6.4 \mathrm{keV}$ Fe-K-alpha line (observed at $\mathrm{E}_{\text {obs }}=2.738 \mathrm{keV}$ ) with the limits on equivalent width given as $\mathrm{EW}<750 \mathrm{eV}$ and $\mathrm{EW}<82 \mathrm{eV}$, respectively. However, the narrow line was detected in Haro-Corzo et al. (2007) analysis of one Chandra data at $\mathrm{E}_{\mathrm{obs}}=2.84 \mathrm{keV}$. Here we present all available Chandra observations and search for the

\footnotetext{
8 http://cxc.harvard.edu/cda.
} 
Table 2: Description of the Chandra Observations for PG1634+706

\begin{tabular}{ccc}
\hline \hline Observed data set & Exposure time (sec.) & Total counts \\
\hline obs-id 47 & 5389.08 & 1651 \\
obs-id 62 & 4854.57 & 1472 \\
obs-id 69 & 4859.42 & 1457 \\
obs-id 70 & 4859.68 & 1419 \\
obs-id 71 & 4405.57 & 1356 \\
obs-id 1269 & 10834.03 & 2216 \\
\hline \hline
\end{tabular}

narrow emission line within the available energy range.

PG1634+706 was observed with Chandra ACIS-S detector (Weisskopf et al. 2002) as a calibration target six times on March 23 and 24, 2000. Each observation lasted between 4.4 and $11 \mathrm{ksec}$. We use CIAO softwareh to process the archival data and extracted the spectra assuming circular source regions of 1.8 arcsec radius. We apply CALDB 3.3.0 calibration data. Table 2 lists each observation with its exposure time and the total counts in its spectrum.

Below we apply our methods and search for the narrow emission line in the available Chandra spectra.

\section{2. $\quad$ Fitting a Spectral Model}

We use our statistical algorithms using a delta function line profile to search for a line in the Chandra spectra of PG1634+706. When we look for emission lines, we typically confine our attention to energies above $1 \mathrm{keV}$ because we avoid regions with potential calibration issues and effects related to absorption.

The marginal posterior distribution of the line location for each observation of PG1634+706 is computed using Monte Carlo draws and is presented in the top two rows of Figure 9, The solid lines represent the marginal posterior distributions, and the corresponding profile posterior distributions are represented by dashed lines. Although the marginal and profile posterior distributions differ in their treatment of nuisance parameters, Figure 9 illustrates

\footnotetext{
h http://cxc.harvard.edu/ciao.
} 

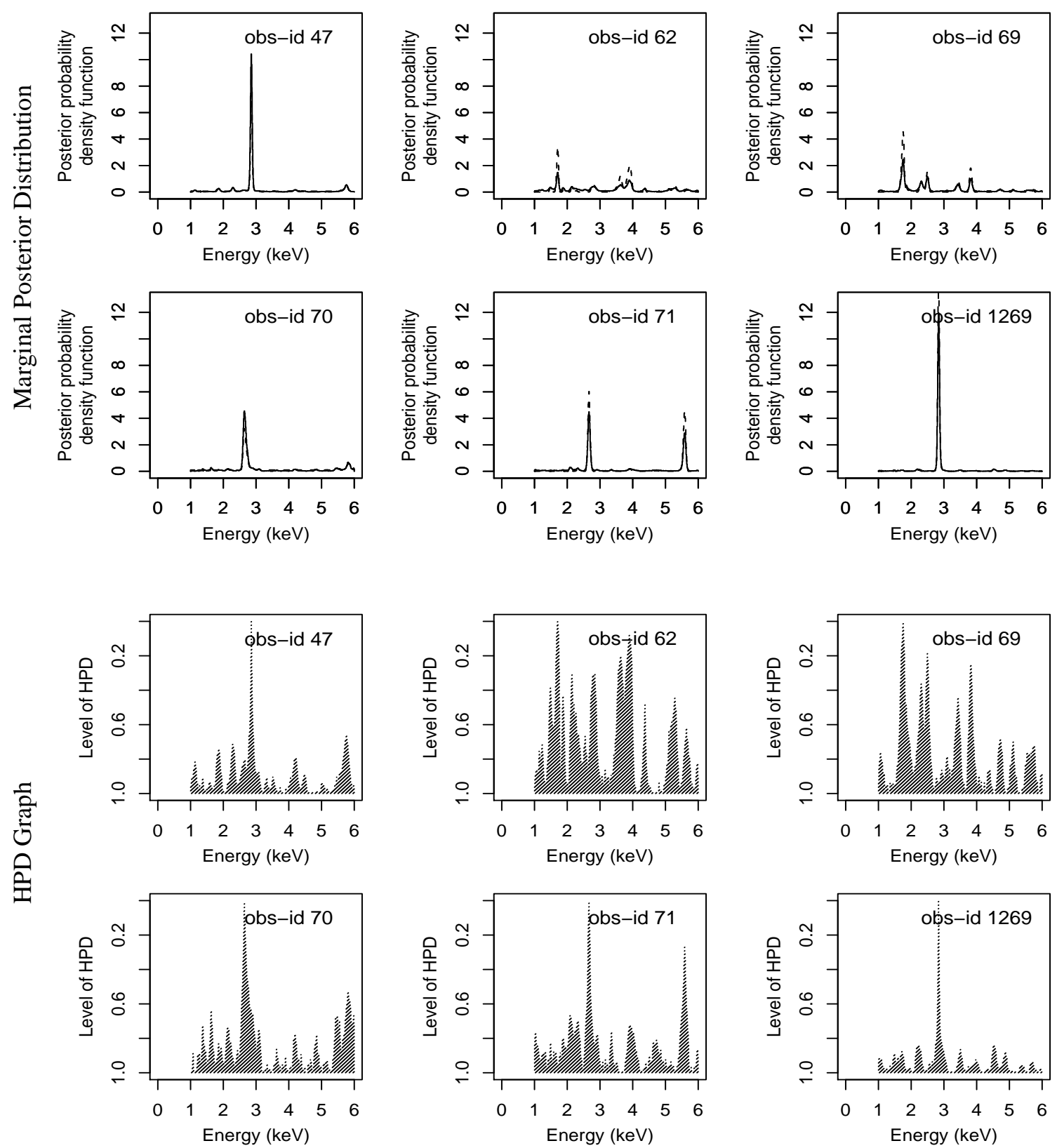

Fig. 9.- The Marginal Posterior Distribution and HPD Graph of the Delta Function Line Location, $\mu$, for Each of the Six Observations of PG1634+706. The solid lines in the first two rows represent the marginal posterior distribution of the delta function line location, and the dashed lines the profile posterior distribution. For several of the observations the two are nearly indistinguishable. 
Table 3: 95\% HPD Regions of the Delta Function Line Location. The posterior modes of the line location near $2.74 \mathrm{keV}$ where the Fe-K-alpha emission line is identified are indicated in bold face.

\begin{tabular}{cccc}
\hline \hline $\begin{array}{c}\text { Observed } \\
\text { data set }\end{array}$ & $\begin{array}{c}\text { Posterior } \\
\text { mode }(\mathrm{keV})\end{array}$ & $\begin{array}{c}\text { 95\% HPD } \\
\text { region (keV) }\end{array}$ & $\begin{array}{c}\text { Posterior } \\
\text { probability }\end{array}$ \\
\hline \multirow{2}{*}{ obs-id 47} & $\mathbf{2 . 8 8 5}$ & $(2.44,3.14)$ & $72.48 \%$ \\
& 5.915 & $(5.44,5.92)$ & $8.48 \%$ \\
\hline \multirow{4}{*}{ obs-id 62} & 1.885 & $(1.00,1.97)$ & $25.88 \%$ \\
& $\mathbf{2 . 7 8 5}$ & $(2.05,3.02)$ & $21.65 \%$ \\
& 3.925 & $(3.30,4.06)$ & $28.27 \%$ \\
& 5.395 & $(4.99,5.41)$ & $8.17 \%$ \\
\hline \multirow{2}{*}{ obs-id 69} & 1.955 & $(1.51,2.65)$ & $55.75 \%$ \\
& 3.535 & $(2.84,3.62)$ & $12.41 \%$ \\
\hline \multirow{2}{*}{ obs-id 70 } & 3.935 & $(3.70,4.01)$ & $11.79 \%$ \\
\hline \multirow{3}{*}{ obs-id 71 } & $\mathbf{2 . 7 9 5}$ & $(2.37,3.17)$ & $63.22 \%$ \\
& 5.945 & $(5.34,6.00)$ & $15.96 \%$ \\
\hline obs-id 1269 & 2.325 & $(1.75,2.45)$ & $8.81 \%$ \\
\hline \hline
\end{tabular}

that both representations capture similar peaks and confirms that the Markov chain fully explores the parameter space for the delta function line location. Because the marginal posterior distribution of the delta function line location is highly multimodal, we summarize the posterior distribution by constructing an HPD graph to visualize the HPD regions of varying levels; see $\$ 2.3$. The HPD graph also illustrates that the marginal posterior distribution is highly multimodal, so that each HPD region may consists of a number of disjoint intervals.

For example, the 95\% HPD regions of the delta function line location are presented in Table 3 along with local modes of the posterior distribution associated with each interval. Each of the 95\% HPD regions is composed of a number of disjoint intervals. Only the intervals that have posterior probabilities greater than $5 \%$ are presented in Table 3 , so that the probabilities may sum to less than $95 \%$. For example, the two intervals of obs-id 47

\footnotetext{
${ }^{\mathrm{i}}$ Note that the posterior probability combined for each obs-id may not add up to $95 \%$ because we list only posterior intervals with posterior probability of $5 \%$ or more.
} 

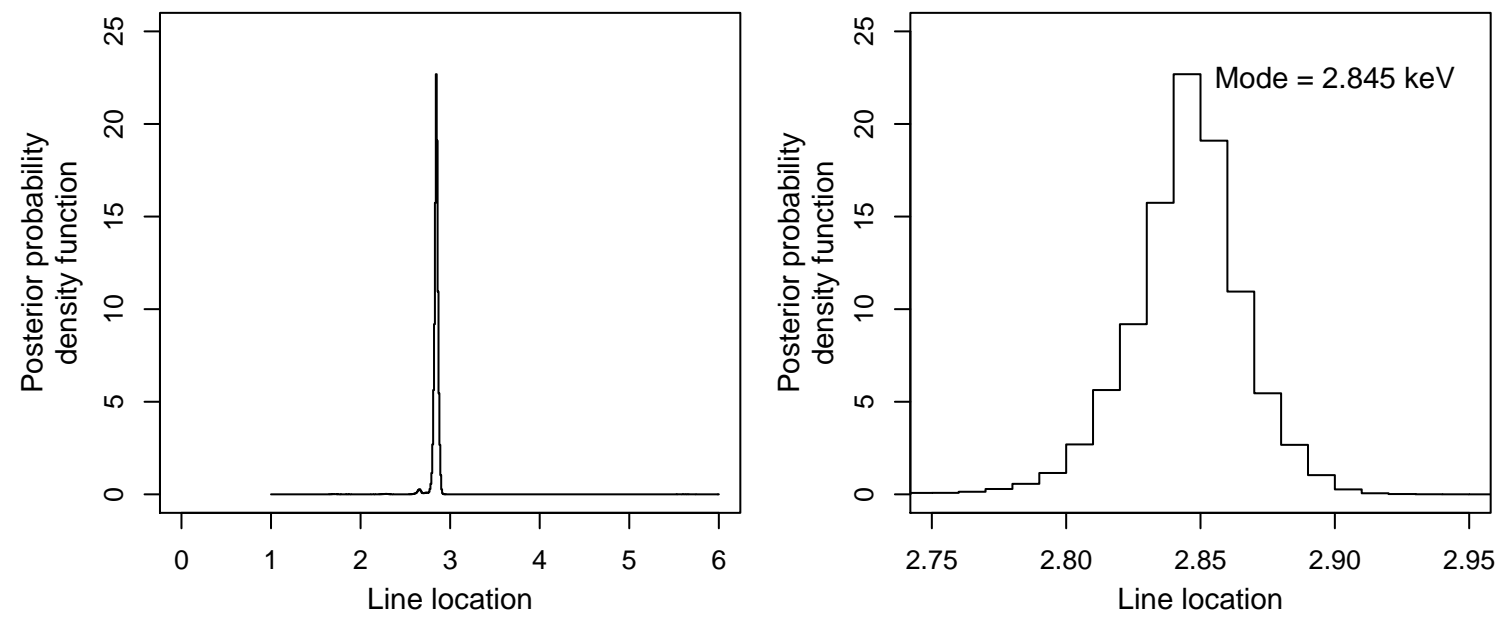

Fig. 10.- Posterior Distribution of the Line Location Given All of the Observations of PG1634+706. The left panel is plotted over the entire energy range of $\mu$ and the right panel over a range near $2.85 \mathrm{keV}$.

presented in Table 3 have a combined posterior probability of $80.96 \%$ and the other eleven intervals not shown in the table have a posterior probability of about $14.04 \%$, for a total of $95 \%$. The posterior modes of the delta function line location that are located near $2.74 \mathrm{keV}$ are indicated in bold face in Table 3 .

The six observations of PG1634+706 were independently observed with Chandra. Thus, under the flat prior distribution on $\mu, p(\mu) \propto 1$, the posterior distribution of the line location given all six observations is given by

$$
\begin{aligned}
p\left(\mu \mid \mathbf{Y}^{\mathrm{obs}}\right) & \propto \int \cdots \int \prod_{i=1}^{6} L\left(\mu, \psi_{i} \mid \mathbf{Y}_{i}^{\mathrm{obs}}\right) d \psi_{1} \cdots d \psi_{6} \\
& =\prod_{i=1}^{6} p\left(\mu \mid \mathbf{Y}_{i}^{\mathrm{obs}}\right)
\end{aligned}
$$

where $\mathbf{Y}^{\mathrm{obs}}=\left\{\mathbf{Y}_{i}^{\mathrm{obs}}, i=1, \ldots, 6\right\}$ denotes the six observations, $\mu$ denotes the delta function line location parameter, $\psi=\left\{\psi_{i}, i=1, \ldots, 6\right\}$ denotes the set of model parameters other than $\mu$ for the six observations, and $L\left(\mu, \psi_{i} \mid \mathbf{Y}^{\text {obs }}\right)$ represents a likelihood function of $\left(\mu, \psi_{i}\right)$ given $\mathbf{Y}^{\text {obs }}$. (Here we allow $\psi_{i}$ to vary among the six observations; i.e., we do not exclude the possibility that the six observations have somewhat different power law normalizations and photon indexes.) The values of the posterior distribution given one of the individual data sets is sometimes indistinguishable from zero because of numerical inaccuracies. Thus 
we add $1 / 15000$ to the posterior probability of each energy bin and renormalize each of the posterior distributions. This allows the product given in Equation 6 to be computed for each energy bin and is somewhat conservative as it increases the posterior uncertainty corresponding to each of the individual data sets. Figure 10 presents the marginal posterior distribution of the delta function line location given all six observations computed in this way; the left panel examines the whole range of the line location while the right panel focuses on the range near $2.74 \mathrm{keV}$. As shown in Figure 10, the posterior distribution given all six observations is fairly unimodal and symmetric, except the little local mode near $2.655 \mathrm{keV}$. Thus, using a nominal 95\% HPD region, the most probable delta function line location is summarized as $2.845_{-0.055}^{+0.045} \mathrm{keV}$ with posterior probability of $95.3 \%$.

\subsection{Model Checking and Evidence for the Emission Line}

Posterior predictive methods (Rubin 1981, 1984; Meng 1994; Gelman \& Meng 1996; Gelman et al. 1996) can be employed to check the specification of the spectral model. This methods aim to check the self-consistency of a model, i.e., the ability of the fitted model to predict the data to which the model is fit. To evaluate and quantify evidence for the inclusion of an emission line in the spectrum, we extend the method of posterior predictive p-values proposed by Protassov et al. (2002) and van Dyk \& Kang (2004).

With the Chandra observations of PG1634+706, we consider the same spectral model discussed in $\$ 1.3$ except that we compare three models for the emission line:

Model 0 : There is no emission line in the spectrum.

Model 1 : There is a delta function emission line with location fixed at $2.74 \mathrm{keV}$ but unknown intensity in the spectrum.

Model 2 : There is a delta function emission line with unknown location and intensity in the spectrum.

We could equally well consider a Gaussian line profile in Models 1 and 2; either line profile model results in a valid test. We consider a delta function line profile simply because we are looking for evidence of a narrow emission line.

We use ppp-values to compare the three models and quantify the evidence in the data for

the delta function emission line; see Protassov et al. (2002) for details of this method and its advantages over the standard F-test, the standard Cash statistic (Cash 1979) (or likelihood 

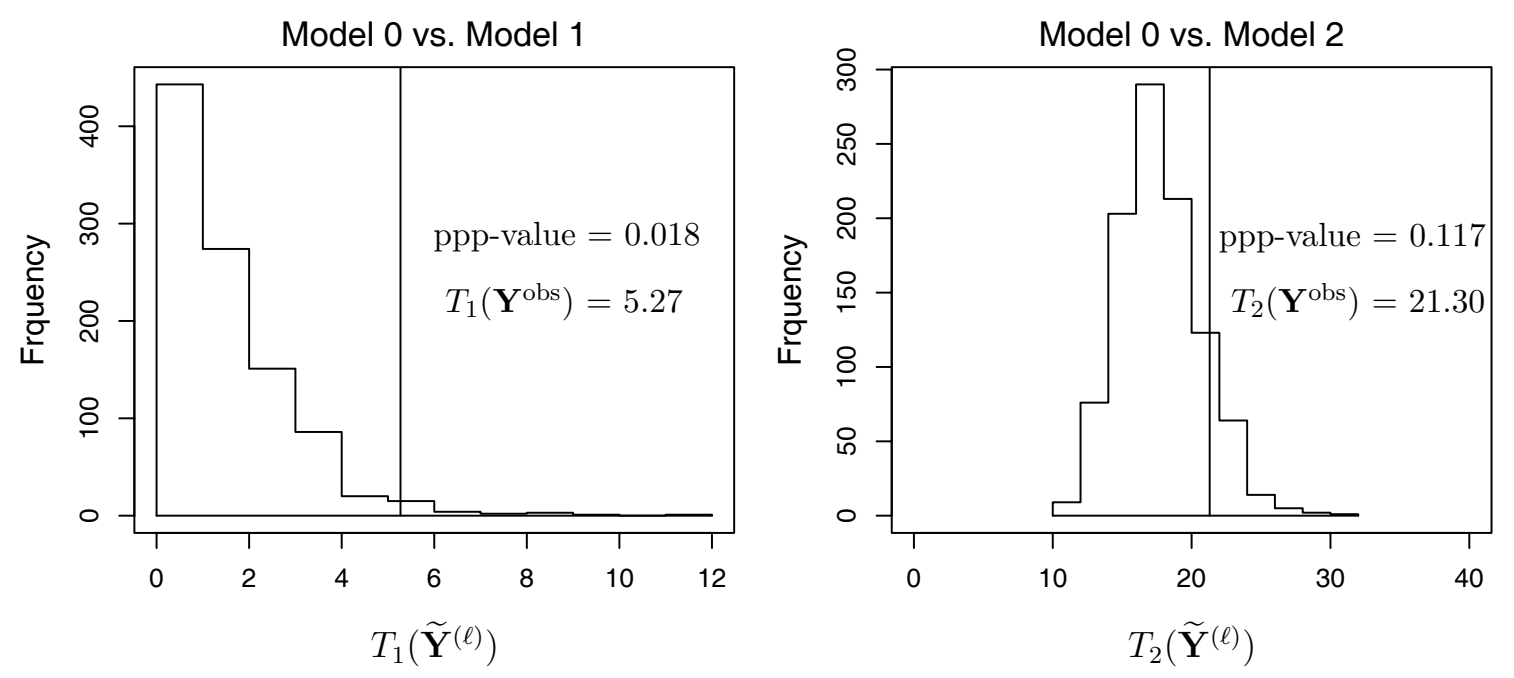

Fig. 11. - Posterior Predictive Checks Given All Six Observations of PG1634+706. In each of the two histograms, the observed test statistic (the vertical line) is compared with the test statistics from 1000 posterior predictive simulated data sets. The ppp-value is the proportion of the test statistics computed using the data simulated under Model 0 that are as extreme as or more extreme than the observed test statistic. Small ppp-values indicate stronger evidence for the emission line.

ratio test statistic), and Bayes Factordj. In the posterior predictive checks, Model 1 fixes the delta function line location at $2.74 \mathrm{keV}$ using prior information as to the location of the Fe-K-alpha emission line. In order to combine the evidence for the line from all six observations with different exposure areas and exposure times, we base our comparisons on the test statistic that is the sum of the six loglikelihood ratio statistics for comparing Model $m$ and Model 0, i.e.,

$$
T_{m}\left(\tilde{\mathbf{Y}}^{(\ell)}\right)=\sum_{i=1}^{6} \ln \left\{\frac{\sup _{\theta \in \Theta_{m}} L\left(\theta \mid \widetilde{\mathbf{Y}}_{i}^{(\ell)}\right)}{\sup _{\theta \in \Theta_{0}} L\left(\theta \mid \widetilde{\mathbf{Y}}_{i}^{(\ell)}\right)}\right\}, \quad m=1,2, \text { and } \ell=1, \ldots, 1000
$$

where $\Theta_{0}, \Theta_{1}$, and $\Theta_{2}$ represent the parameter spaces under Models 0,1 , and 2, respectively, and $\tilde{\mathbf{Y}}^{(\ell)}=\left\{\tilde{\mathbf{Y}}_{i}^{(\ell)}, i=1, \ldots, 6\right\}$ denotes the collection of six data sets simulated under

\footnotetext{
${ }^{j}$ Because we are looking for evidence for the emission line, we do not treat the two models under comparison equally. That is, we are not simply looking for the model (with or without the emission line) that best explains the data but we are also attempting to guard against wrongly concluding that an emission line is present when there is none. If we wished to simply compare the two models, the deviance information criterion (DIC, Spiegelhalter et al. 2002) would be a better method to use.
} 
Model 0. Specifically, we generate 1000 replications of each data set from the posterior predictive distribution under Model 0 and compute $T_{m}\left(\widetilde{\mathbf{Y}}^{(\ell)}\right)$ for $m=1,2$. Histograms of $T_{1}\left(\widetilde{\mathbf{Y}}^{(\ell)}\right)$ and $T_{2}\left(\widetilde{\mathbf{Y}}^{(\ell)}\right)$ appear in Figure 11, Comparing the histogram of the simulated test statistics with the observed value of the test statistic yields the ppp-values (Rubin 1984; Meng 1994) shown in Figure 11. The ppp-value is the proportion of the simulated test statistics that are as extreme as or more extreme than the observed test statistic. Smaller ppp-values give stronger evidence for the alternative model, i.e., Model 1 or Model 2 , thereby supporting the inclusion of the line in the spectrum in our case. As shown in Figure 11, there is evidence for the presence of the spectral line given all six observations. The comparison between Models 0 and 1 shows stronger evidence for the line location because we are using extra a priori information about the plausible line location.

\section{Concluding Remarks}

This article presents methods to detect, identify, and locate narrow emission lines in Xray spectra via a highly structured multilevel spectral model that includes a delta function line profile. Modeling narrow emission lines with a delta function causes the EM algorithms and MCMC samplers developed in van Dyk et al. (2001) to break down and thus requires more sophisticated statistical methods and algorithms. The marginal posterior distribution of the delta function emission line location tends to be highly multimodal when the emission line is weak or multiple emission lines are present in the spectrum. Because basic summary statistics are not appropriate to summarize such a multimodal distribution, we instead develop and use HPD graphs along with a list of posterior modes. Testing for an emission line in the spectrum is a notoriously challenging problem because the value of the line intensity parameter is on the boundary of the parameter space, i.e., zero, under a model that does not include an emission line. Thus, we extend the posterior predictive methods proposed by Protassov et al. (2002) to test for the evidence of a delta function emission line with unknown location in the spectrum.

Using the simulation study in \$3, we demonstrate the potential advantage of model misspecification using a delta function line profile in place of a Gaussian line profile. We show that the delta function line profile may provide more precise and meaningful summaries for line locations if the true emission line is narrow. When multiple lines are present in the spectrum, the marginal posterior distribution of a single delta function line location may indicate multiple lines in the spectrum.

Our methods are applied to the six different Chandra observations of PG1634+706 in order to identify a narrow emission line in the X-ray spectrum. Given all the six observations, 
the most probable delta function line is identified at $2.845_{-0.055}^{+0.045} \mathrm{keV}$ in the observed frame. The corresponding rest frame energy for the line is $6.64_{-0.13}^{+0.11} \mathrm{keV}$, which may suggest the high ionization of iron in the emitting plasma. There is some recent evidence that high ionization iron line can be variable on short timescales (see for example Mkn766 in Miller et al. 2006). Such variability would explain no detection of the emission line in one of the six Chandra observations.

The authors gratefully acknowledge funding for this project partially provided by NSF grant DMS-04-06085 and by NASA Contract NAS8-39073 and NAS8-03060 (CXC). This work is a product of joint work with the California-Harvard astrostatistics collaboration (CHASC) whose members include J. Chiang, A. Connors, D. van Dyk, V. L. Kashyap, X.L. Meng, J. Scargle, A. Siemiginowska, E. Sourlas, T. Park, A. Young, Y. Yu, and A. Zezas. The authors also thank the referee for many helpful and constructive comments.

\section{A. New Algorithms for Mode Finding and Posterior Simulation}

In this section we give an overview of the mode finding and posterior simulation methods used to fit the spectral model with a narrow emission line. Our summary is brief, and thus readers who are interested in a more detailed description should refer to van Dyk \& Park (2004, 2008) and Park \& van Dyk (2008).

\section{A.1. Faster EM-type Algorithms for Mode Finding}

In order to illustrate our computational strategy, consider a simplified example of an ideal instrument that is not subject to the data contamination processes. In particular, the redistribution matrix is an identity matrix, the effective area is constant, there are no absorption features, and there is no background contamination. In addition, we assume that the continuum is specified with no unknown parameters and that there is a single Gaussian emission line that has a known width $\nu_{0}$ and a known intensity $\lambda$. Thus, the line location is the only unknown parameter, the source model given in Equation 1 simplifies to

$$
\Lambda_{j}(\mu)=\Delta_{j} f\left(E_{j}\right)+\lambda \pi_{j}\left(\mu, \nu_{0}\right)
$$

and the counts are modeled as $Y_{j} \sim \operatorname{Poisson}\left(\Lambda_{j}(\mu)\right)$. This model can be fit using the method

of data augmentation (Tanner \& Wong 1987) by setting $Y_{j}=Y_{j}^{C}+Y_{j}^{L}$, where $Y_{j}^{C}$ and $Y_{j}^{L}$ are the counts due to the continuum and the emission line in bin $j$, respectively. In particular, 
the EM iteratively split the counts into continuum counts and emission line counts. Given the current iterate of the line location, $\mu^{(t)}$, the E-STEP updates the line counts via

E-STEP : Compute $\mathrm{E}\left[Y_{j}^{L} \mid \mu^{(t)}, \mathbf{Y}\right]$ for each bin $j$. That is,

$$
\widehat{Y}_{j}^{L} \equiv \mathrm{E}\left[Y_{j}^{L} \mid \mu^{(t)}, \mathbf{Y}\right]=Y_{j} \frac{\lambda \pi_{j}\left(\mu^{(t)}, \nu_{0}\right)}{\Delta_{j} f\left(E_{j}\right)+\lambda \pi_{j}\left(\mu^{(t)}, \nu_{0}\right)} .
$$

Next, the M-sTEP of EM updates the emission line location $\mu^{(t+1)}$ by

M-STEP : Find $\mu^{(t+1)}=\sum_{j \in \mathcal{J}} E_{j} \widehat{Y}_{j}^{L} / \sum_{j \in \mathcal{J}} \widehat{Y}_{j}^{L}$

which is the weighted average of the bin energies and uses the emission line counts as weights. Although this EM algorithm is simple, it breaks down when fitting the location of a narrow emission line, i.e., when $\nu_{0}$ is small relative to the size of the bins. In the extreme, the Gaussian line profile becomes a delta function, so that $\pi_{j}\left(\mu^{(t)}, \nu_{0}\right)$ is zero for all bins except the bin containing $\mu^{(t)}$. This results in an E-step that computes zero line counts in all bins except the bin containing $\mu^{(t)}$ and finally an M-step that computes $\mu^{(t+1)}=\mu^{(t)}$. This means that EM will return the same line location at each iteration and that the algorithm will not converge to a mode.

In this simplified example, this difficulty can be avoided by directly maximizing the posterior distribution

$$
p(\mu \mid \mathbf{Y}) \propto \prod_{j \in \mathcal{J}}\left\{\Lambda_{j}(\mu)\right\}^{Y_{j}} e^{-\Lambda_{j}(\mu)} .
$$

Because of the binning of the data, we can treat possible line locations within each bin as indistinguishable and compute the mode by evaluating Equation A3 on the fine grid that corresponds to the binning of the data.

The situation is more complicated in the full spectral model described in \$1.3. The method of data augmentation can be used to construct efficient algorithms that both fit the parameters in the continuum and the lines and account for instrument response and background contamination. In the case of the narrow emission line, however, we must implement a strategy that uses less data augmentation when updating the line location/width than when updating the other model parameters. The Expectation/Conditional Maximization Either (ECME) algorithm (Liu \& Rubin 1994) allows us to use no data augmentation when updating the line location/width, but the resulting M-step is time consuming owing to the multiple evaluations of the conditional posterior distribution of the line location/width 
which involve the large dimensional redistribution matrix, M. An intermediate strategy uses the standard data augmentation scheme to adjust for instrument response and background contamination but does not separate continuum and line photons when updating the line location/width. This strategy is an instance of the Alternating Expectation/Conditional Maximization (AECM) algorithm (Meng \& van Dyk 1997) and each iteration is much quicker than with ECME but more iterations are required for convergence. The algorithms we use for mode finding aim to combine the advantages of ECME and AECM by running one ECME iteration followed by $m$ AECM iterations and repeating until convergence. van Dyk \& Park (2004) call this a Rotation $(m)$ algorithm and illustrate the computational advantage of the strategy.

\section{A.2. Faster MCMC Samplers for Posterior Simulation}

Returning to the simplified example of Appendix A.1, we can formulate a Gibbs sampler using the same data augmentation scheme. Given the current iterate, $\mu^{(t)}$, SteP 1 simulates the line counts in bin $j$ via

STEP 1 : Simulate $\left(Y_{j}^{L}\right)^{(t+1)}$ from $p\left(Y_{j}^{L} \mid \mu^{(t)}, \mathbf{Y}\right)$ for $j=1, \ldots, J$. That is,

$$
Y_{j}^{L} \mid\left(\mu^{(t)}, \mathbf{Y}\right) \sim \operatorname{Binomial}\left(Y_{j}, \frac{\lambda \pi_{j}\left(\mu^{(t)}, \nu_{0}\right)}{\Delta_{j} f\left(E_{j}\right)+\lambda \pi_{j}\left(\mu^{(t)}, \nu_{0}\right)}\right) .
$$

Next, Step 2 simulates the line location via

STEP 2 : Simulate $\mu^{(t+1)}$ from $p\left(\mu \mid\left(\mathbf{Y}^{L}\right)^{(t+1)}, \mathbf{Y}\right)$. That is,

$$
\mu \mid\left(\left(\mathbf{Y}^{L}\right)^{(t+1)}, \mathbf{Y}\right) \sim \mathrm{N}\left(\frac{\sum_{j \in \mathcal{J}} E_{j}\left(Y_{j}^{L}\right)^{(t+1)}}{\sum_{j \in \mathcal{J}}\left(Y_{j}^{L}\right)^{(t+1)}}, \frac{\nu_{0}^{2}}{\sum_{j \in \mathcal{J}}\left(Y_{j}^{L}\right)^{(t+1)}}\right) .
$$

We collect a posterior sample $\left\{\mu^{(t)}, t=t_{0}+1, \ldots, T\right\}$ after a sufficiently long burn-in period $t_{0}$; we discard the burn-in draws, see van Dyk et al. (2001) for details.

When this algorithm is applied with a narrow emission line, it breaks down just as the EM algorithm does. When a delta function is used for the line profile, the simulation in Equation A4 results in no line counts in any bin except the one containing the line, and again

$\mu$ does not move from its starting value. When a narrow Gaussian line profile is used, the situation is less extreme, but the sampler exhibits very high autocorrelations and typically 
cannot jump among the posterior modes. Just as with EM, the difficulty can be avoided by computing the posterior distribution of $\mu$ on a find grid and directly simulating

$$
\mu^{(t)} \sim \operatorname{Multinomial}\left\{1 ;\left\{\left.p(\mu \mid \mathbf{Y})\right|_{\mu=E_{j}}, j \in \mathcal{J}\right\}\right\}
$$

When the method of data augmentation is used to account for data contamination processes described in $\$ 1.3$, however, this approach should be modified in a manner analogous to the ECME and AECM algorithms. This leads to the strategy of using conditional distributions from different data augmentation schemes. In this case, however, the resulting set of conditional distributions used to construct the Gibbs sampler may be incompatible and there may be no joint distribution that corresponds to this set of conditional distributions. Although such a sampler may result in efficient computation, care must be taken to be sure the sampler delivers simulations from the target posterior distribution. This is formalized through the Partially Collapsed Gibbs (PCG) sampler of van Dyk \& Park (2008) which outlines the steps that should be taken to ensure proper convergence; refer to Park \& van Dyk (2008) for the applications and illustrations of PCG samplers. The PCG samplers can be viewed as the stochastic version of ECME and AECM, thereby allowing us to sample the line location with no data augmentation (as in ECME) or partial data augmentation (as in AECM). Thus, the PCG sampler differs from the Gibbs sampler developed in van Dyk et al. (2001) in sampling the line location (and line width), and in the order of sampling steps. Park \& van Dyk (2008) design two PCG samplers to fit the spectral model with a delta function emission line or a narrow Gaussian emission line, which are called PCG I and PCG II, respectively. As compared to PCG I, PCG II requires one additional sampling step

for the line width, so that fitting the narrow Gaussian emission line is computationally more demanding than fitting the delta function emission line.

\section{REFERENCES}

Cash, W. 1979, ApJ, 228, 939

Chartas, G., Brandt, W. N., Gallagher, S. C., \& Garmire, G. P. 2002, ApJ, 579, 169

Critchley, F., Ford, I., \& Rijal, O., 1988, Biometrika, 75, 21.

Dempster, A. P., Laird, N. M., \& Rubin, D. B., 1977, JRSS, 39, 1.

Fabian, A. C. 2006, The X-ray Universe 2005, 604, 463 (astro-ph/0511537)

Freeman, P., Doe, S., \& Siemiginowska, A., 2001, Proceedings of SPIE, 4477, 76. 
Gallagher, S. C., Brandt, W. N., Chartas, G., \& Garmire, G. P., 2002, ApJ, 567, 37.

Gelman, A., Carlin, J., Stern, H., \& Rubin, D. B., 1995, Chapman \& Hall/CRC, New York.

Gelman, A. \& Meng, X.-L., 1996, MCMC in Practice, Chapman \& Hall, New York.

Gelman, A., Meng, X.-L., \& Stern, H., 1996, Statistica Sinica, 6, 733.

Gelman, A. \& Rubin, D. B., 1992, Statistical Science, 7, 457.

Geman, S. \& Geman, D., 1984, IEEE Transactions on PAMI, 6, 721.

George, I. M., Turner, T. J., Yaqoob, T., Netzer, H., Laor, A., Mushotzky, R. F., Nandra, K., \& Takahashi, T. 2000, ApJ, 531, 52

Guainazzi, M., Bianchi, S., \& Dovčiak, M. 2006, Astronomische Nachrichten, 327, 1032

Haro-Corzo, S. A. R., Binette, L., Krongold, Y., Benitez, E., Humphrey, A., Nicastro, F., \& Rodríguez-Martínez, M. 2007, ApJ, 662, 145

Jiang, P., Wang, J. X., \& Wang, T. G. 2006, ApJ, 644, 725

Jiménez-Bailón, E., Piconcelli, E., Guainazzi, M., Schartel, N., Rodríguez-Pascual, P. M., \& Santos-Lleó, M. 2005, A\&A, 435, 449

Lampton, M., Margon, B., \& Bowyer, S., 1976, ApJ, 208, 177.

Liu, C. \& Rubin, D. B., 1994, Biometrika, 81, 633.

Markoff, S., Nowak, A. N., \& Wilms, J., 2005, ApJ, 635, 1203.

Meng, X.-L., 1994, The Annals of Statistics, 22, 1142.

Meng, X.-L. \& van Dyk, D. A., 1997, JRSS, 59, 511.

Miller, J. M. 2006, Astronomische Nachrichten, 327, 997

Miller, L., Turner, T. J., Reeves, J. N., George, I. M., Porquet, D., Nandra, K., \& Dovciak, M. 2006, A\&A, 453, L13

Miller, J. M. 2007, ARA\&A, 45, 441

Miller, L., Turner, T. J., \& Reeves, J. N. 2008, A\&A, 483, 437

Nandra, K., George, I. M., Mushotzky, R. F., Turner, T. J., \& Yaqoob, T. 1997, ApJ, 488, L91 
Park, T. \& van Dyk, D. A., 2008, Tech. Rep.

Page, K. L., Reeves, J. N., O’Brien, P. T., \& Turner, M. J. L. 2005, MNRAS, 364, 195

Pounds, K. A., \& Reeves, J. N. 2007, MNRAS, 374, 823

Press, W., Flannery, B., Teukolsky, S., \& Vetterling, W., 1992, Cambridge University Press.

Protassov, R., et al., 2002, ApJ, 571, 545.

Rees, M. J., 1978, Physica Scripta, 17, 193.

Reeves, J. N., et al. 2006, Astronomische Nachrichten, 327, 1079

Rubin, D. B., 1981, Journal of Educational Statistics, 6, 377.

Rubin, D. B., 1984, The Annals of Statistics, 12, 1151.

Sikora, M., Madejski, G., Moderski, R., \& Poutanen, J., 1997, ApJ, 484, 108.

Sobolewska, M. A., Siemiginowska, A., \& Zycki, P. T., 2004, ApJ, 617, 102.

Spiegelhalter, D. J., Best, N. G., Carlin, B. P., \& van der Linde, A., 2002, JRSS, 64, 583.

Steidel, C. C. \& Sargent, W. L. W., 1991, ApJ, 382, 433.

Tanner, M. A. \& Wong, W. H., 1987, JASA, 82, 528.

van Dyk, D. A., et al., 2006, Bayesian Analysis, 1, 139.

van Dyk, D. A. \& Park, T., 2004, Wiley \& Sons, New York.

van Dyk, D. A. \& Park, T., 2008, JASA, To appear.

van Dyk, D. A., Connors, A., Kashyap, V., \& Siemiginowska, A., 2001, ApJ, 548, 224.

van Dyk, D. A. \& Hans, C. M., 2002, Spatial Cluster Modelling, 175, CRC Press, London. van Dyk, D. A. \& Kang, H., 2004, Statistical Science, 19, 275.

Venzon, D. J. \& Moolgavkar, S. H., 1988, Applied Statistics, 37, 87.

Weisskopf, M. C., et al., 2002, PASP 114, 1.

Yaqoob, T., George, I. M., Nandra, K., Turner, T. J., Serlemitsos, P. J., \& Mushotzky, R. F. 2001, ApJ, 546, 759 
Yaqoob, T., Padmanabhan, U., Dotani, T., George, I. M., Nandra, K., Tanaka, Y., Turner, T. J., \& Weaver, K. A. 2001, X-ray Emission from Accretion onto Black Holes,

Yaqoob, T. 2007, Astronomical Society of the Pacific Conference Series, 373, 109 